\title{
THE WAKE DYNAMICS AND FLIGHT FORCES OF THE FRUIT FLY DROSOPHILA MELANOGASTER
}

\author{
MICHAEL H. DICKINSON ${ }^{1}$ AND KARL G. GÖTZ ${ }^{2}$ \\ ${ }^{1}$ Department of Integrative Biology, University of California, Berkeley, CA 94720, USA and \\ ${ }^{2}$ Max-Planck-Institut für Biologische Kybernetik, Spemannstraße 38, D-72076 Tübingen, Germany
}

Accepted 20 May 1996

\begin{abstract}
We have used flow visualizations and instantaneous force measurements of tethered fruit flies (Drosophila melanogaster) to study the dynamics of force generation during flight. During each complete stroke cycle, the flies generate one single vortex loop consisting of vorticity shed during the downstroke and ventral flip. This gross pattern of wake structure in Drosophila is similar to those described for hovering birds and some other insects. The wake structure differed from those previously described, however, in that the vortex filaments shed during ventral stroke reversal did not fuse to complete a circular ring, but rather attached temporarily to the body to complete an inverted heart-shaped vortex loop. The attached ventral filaments of the loop subsequently slide along the length of the body and eventually fuse at the tip of the abdomen. We found no evidence for the shedding of wing-tip vorticity during the upstroke, and argue that this is due to an extreme form of the Wagner effect acting at that time.
\end{abstract}

Summary
The flow visualizations predicted that maximum flight forces would be generated during the downstroke and ventral reversal, with little or no force generated during the upstroke. The instantaneous force measurements using laser-interferometry verified the periodic nature of force generation. Within each stroke cycle, there was one plateau of high force generation followed by a period of low force, which roughly correlated with the upstroke and downstroke periods. However, the fluctuations in force lagged behind their expected occurrence within the wingstroke cycle by approximately $1 \mathrm{~ms}$ or one-fifth of the complete stroke cycle. This temporal discrepancy exceeds the range of expected inaccuracies and artifacts in the measurements, and we tentatively discuss the potential retarding effects within the underlying fluid mechanics.

Key words: vortex wake, insect flight, aerodynamics, Drosophila melanogaster.

\section{Introduction}

As a flying animal moves through the air, it generates aerodynamic forces with its wings and leaves behind a distinct wake. These two processes are inextricably linked, since any aerodynamic force produced by an animal requires an equal and opposite change in fluid momentum. Consequently, the goal of understanding the production of aerodynamic forces may be approached from either of two directions: by studying the time course of force generation by the flapping wings or by characterizing the structure of the resultant wake. These methods are not mutually exclusive, but are instead different approaches to the same problem.

The derivation of mean flight forces from the wake is the underlying basis for the vortex theories of Ellington (1984c) and Rayner $(1979 a, b)$, which both use elaborations of the Rankine-Froude theory of propellers to calculate the steady rate of change of fluid momentum. These theories are simple and robust since they do not require any precise knowledge of the kinematics. By the same token, however, they cannot be used to predict the time history of force generation and offer no direct insight into the underlying aerodynamic mechanisms.
Traditional approaches to the study of aerodynamics focus not on fluid momentum per se, but rather on the means by which the wings generate forces. The basis of the Kutta-Joukowski theory is a direct proportionality between lift and the bound circulation generated by the motion of a wing. It has the crucial disadvantage of being based almost exclusively on steady-state assumptions, which give it limited utility in studies of animal flight. There are, however, an increasing number of theories that consider unsteady mechanisms by which wings generate forces in ways that do not require bound circulation (Maxworthy, 1981; Wu et al. 1991; Spedding, 1992; Dickinson and Götz, 1993; Dickinson, 1994; Ellington, 1995). Together, these theories of airfoil function may eventually offer a framework for assessing the consequences of differences in wing morphology and kinematics - a feature that is missing from approaches that emphasize wake momentum alone.

In spite of the experimental difficulties inherent in visualizing wake structure and making direct measurements of instantaneous flight forces, a cohesive image of force 


\section{M. H. Dickinson AND K. G. GöTZ}

generation and wake structure in birds, bats and insects has begun to emerge. Early flow visualizations confirmed that hovering and slow-flying birds generate a single vortex ring during each wing stroke (Kokshaysky, 1979; Spedding et al. 1984). However, careful quantification of the vortex ring momentum generated by flying birds failed to account for all the forces necessary to sustain flight (Spedding, 1986). This discrepancy may be due to changes in the momentum of the wake that are difficult to visualize. In contrast to the discrete vortex rings produced in hovering flight, the vortex filaments shed from the two wing tips during fast forward flight do not join at the end of the downstroke, but rather give rise to two continuous vortex tubes trailing behind the animal (Rayner et al. 1986; Spedding, 1987). Although many of the earlier wake reconstructions were carried out on birds and bats, a number of exquisite flow visualizations have recently been performed on insects (Brodsky, 1991, 1994; Grodnitsky and Morozov, 1992, 1993). These wake patterns are similar to those of hovering birds, although in some examples the vortex rings of the downstrokes are linked with one another via the tip vortices of a force-generating upstroke, producing a linked vortex chain (Brodsky, 1994). Insects, such as the crane fly Tipula paludosa, with sufficient wing separation during both dorsal and ventral stroke reversal appear to make a separate vortex chain behind each wing (Brodsky, 1991). Owing to limitations in temporal resolution, flow visualization studies have emphasized insects that operate at relatively low wingbeat frequencies. Much less is known of the wake structure in such accomplished fliers as bees, wasps and flies, which employ high wingbeat frequencies. In addition, all the species examined so far fly at relatively high Reynolds numbers. In this paper, we present a wake reconstruction for the fruit fly, Drosophila melanogaster, with a wingbeat frequency of approximately $200 \mathrm{~Hz}$ operating at an intermediate Reynolds number of approximately 100.

Compared with a relatively rich collection of flow visualization studies, calibrated measurements of instantaneous flight forces for flying animals are quite scarce. Cloupeau et al. (1979) compared instantaneous lift measurements in locusts with Jensen's (1956) measurements of lift coefficients on isolated wings and concluded that unsteady mechanisms must augment force production. More recently, Wilkin and colleagues have reached similar conclusions in measurements of instantaneous flight forces of both locusts and moths (Wilkin, 1990, 1991; Wilkin and Williams, 1993). Wolf (1993), although unable to account for inertial forces, has correlated changes in the dynamics of lift within each wing stroke of a locust to changes in the activity of specific flight muscles. Three force recordings have been made using flies (Buckholz, 1981; Zanker and Götz, 1990; G. Nalbach, unpublished results), but these recordings were either uncalibrated or did not account for inertial forces. Although few in number, these experiments provide essential data for testing models of force generation in insect flight.

At some level, the two methods of inquiry, flow visualization and force measurement, must meet to form a cohesive picture. The pressure differential that generates lift according to the Kutta-Joukowski theory is simply another manifestation of the momentum impulse of a growing vortex ring (Milne-Thomson, 1966). The main purpose of this study is to integrate both analyses on the same animal in the hope of constructing a more synthetic view of force generation in flight.

\section{Materials and methods \\ Flow visualization}

The arrangement for visualizing the wake of a tethered fruit fly is indicated in Fig. 1A. Animals were mounted onto a tungsten rod using light-activated cement (GlasKleber, Loctite) and placed within the open working section of a $17 \mathrm{~mm}$ diameter wind-tunnel that produced a laminar flow of $20 \mathrm{~cm} \mathrm{~s}^{-1}$. We aligned the fly within an optical wingbeat analyzer which produced a TTL pulse each stroke during the ventral flip rotation (Götz, 1987; Dickinson et al. 1993). Lycopodium spores, hollow spheres of approximately $30 \mu \mathrm{m}$ diameter, were introduced upstream of the fly within the windtunnel and illuminated with a $0.6 \mathrm{~mm}$ thick sheet of light from a line-generating diode laser $(670 \mathrm{~nm}, 4 \mathrm{~mW})$. The exposure time was controlled by providing current to the laser diode for a specified amount of time. The laser source and linegenerating optics were mounted on a micromanipulator so that the light slice could be moved to illuminate different portions of the wake (Fig. 1B,C). Images of particle trajectories were captured using a Peltier-cooled CCD camera (Photometrics) and stored on a Macintosh Quadra 800 computer for analysis. The Reynolds number for a slowly flying Drosophila is approximately 100 , which is several orders of magnitude below the turbulent transition for a flat plate. For this reason, we expected that all the flows produced by the wings would be laminar.

The animals were tethered in the air flow at a body angle of approximately $20^{\circ}$ with respect to the oncoming flow. The angle of the longitudinal body axis during free flight at an airspeed of $20 \mathrm{~cm} \mathrm{~s}^{-1}$ is expected to be approximately $50^{\circ}$ (see section on force measurements). Therefore, the orientation of the flies in our experiments was not exactly equivalent to free-

Fig. 1. Experimental apparatus for flow visualization and force measurement. (A) The tethered fly flies in the mouth of an open-throat wind-tunnel while Lycopium spores are introduced upstream. (B) Each flow image was exposed by a flash of a laser light triggered by an optical wing sensor (IR LED) that produced a TTL pulse during the ventral flip (Götz, 1987; Dickinson et al. 1993). (C) The camera and laser sheets were arranged to image flow in both sagittal and coronal planes. The sagittal planes labeled A-E, and the coronal planes labeled F-J, correspond roughly to the positions of the optical section shown in Figs 2 and 3. The sagittal plane marked M for midwing corresponds to the optical section in Fig. 4. In the sagittal sections, the laser planes were spaced $1 \mathrm{~mm}$ apart. In the coronal sections, planes F, G, H and I were $0.5 \mathrm{~mm}$ apart, and the distance between planes I and J was $2 \mathrm{~mm}$. (D) Instantaneous flight forces were measured by tracking the displacement of a taut steel wire to which the fly was tethered (see text for details). 
flight conditions. The low body angles at which we tethered the animals in both the flow visualization and interferometry experiments were required in order to maintain the wing stroke plane at an inclination that would produce accurate triggering of the wingbeat analyzer. However, we do not think that this discrepancy is likely to have significant effects on the flow pattern for several reasons. The advance ratio for our tethered flies (calculated according to Ellington, 1984a) was
A
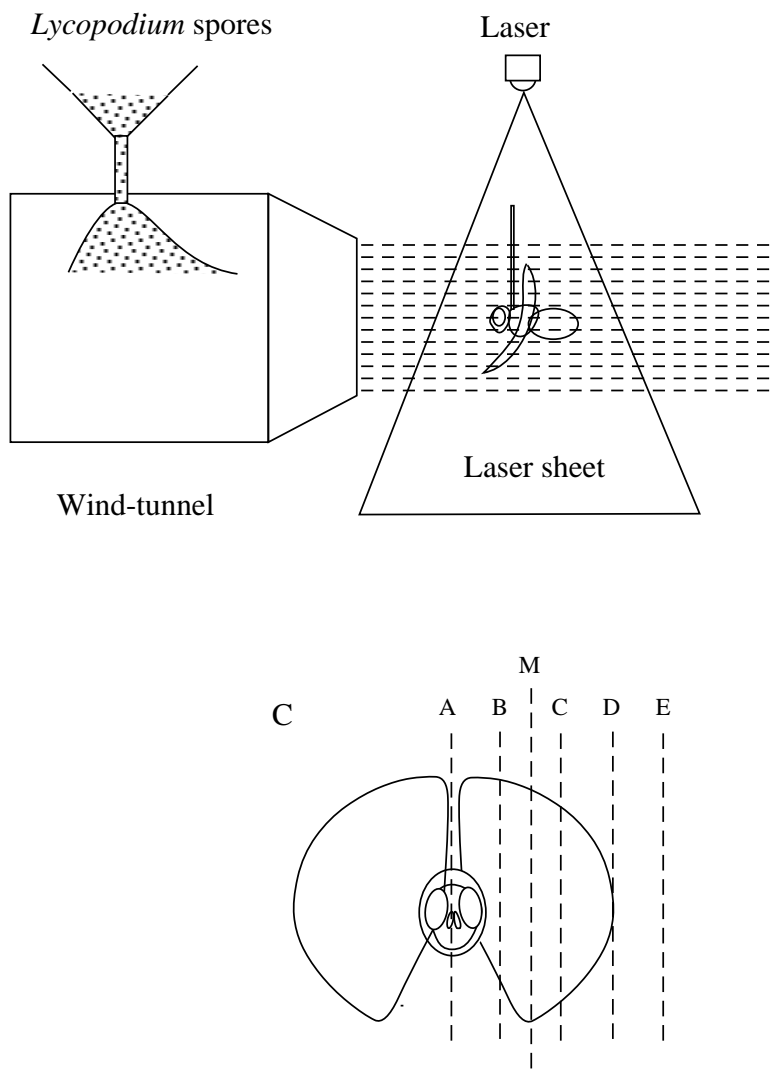

B

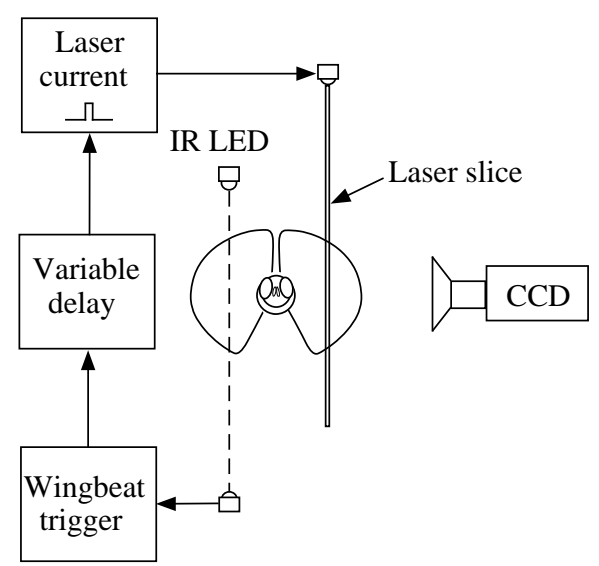

$\mathrm{D}$
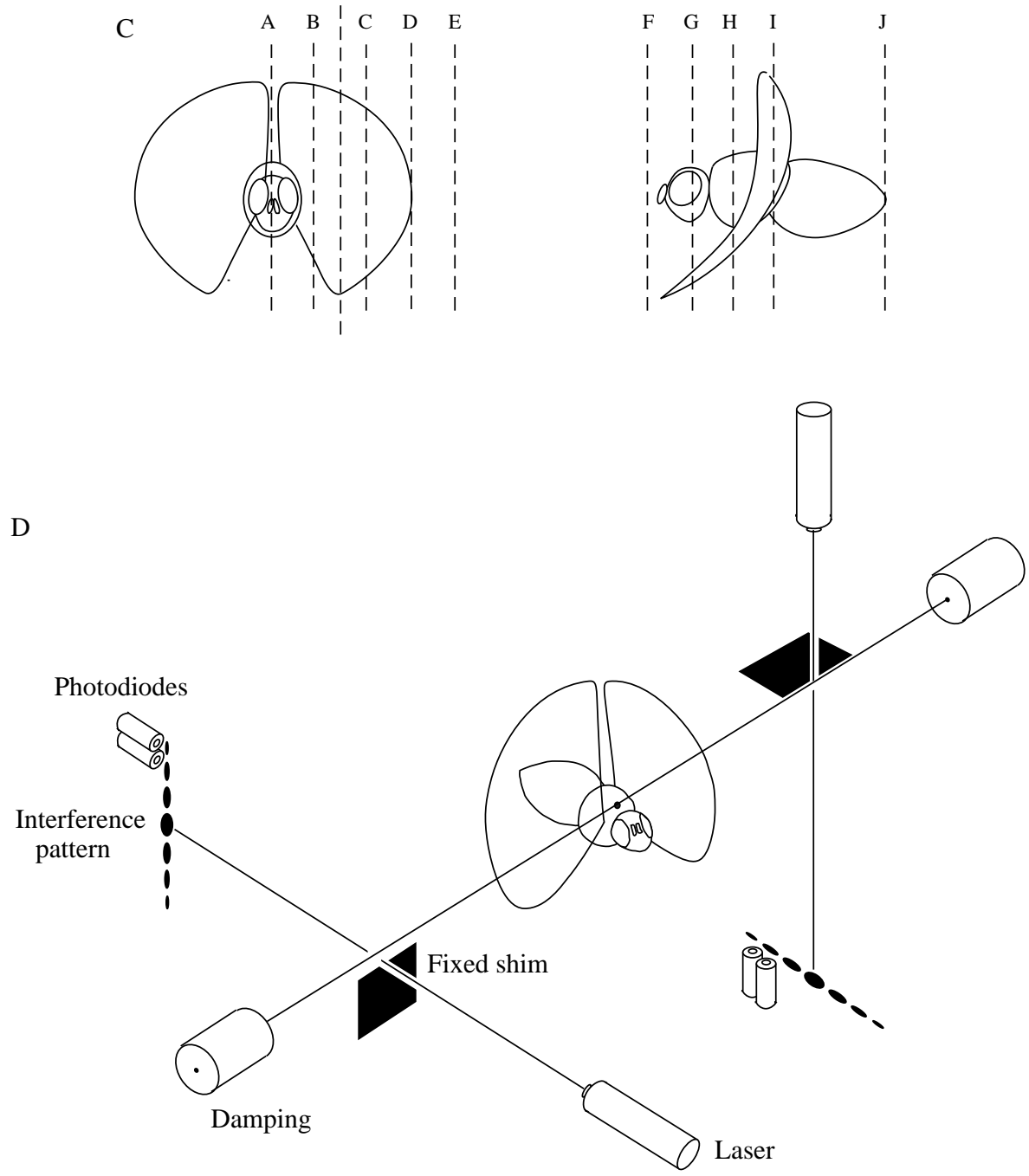

Fig. 1 


\section{M. H. Dickinson AND K. G. GöTZ}

approximately 0.085 , which indicates that the flows are dominated by the beating of the wings and not by the background air velocity of the wind-tunnel. The average sectional wing velocity in Drosophila is $125 \mathrm{~cm} \mathrm{~s}^{-1}$, in a stroke plane that is inclined at approximately $70^{\circ}$ with respect to the body axis. From these values, it is possible to estimate that at a tunnel velocity of $20 \mathrm{~cm} \mathrm{~s}^{-1}$, the $30^{\circ}$ error in the placement of the body angle would cause only a $4 \%$ decrease in the downstroke velocity of the wing and a comparable increase in the upstroke velocity. Further, as described in the Results section, the mean flight forces measured in still and moving air were not statistically different from one another. Thus, despite a body angle that is lower than normal, we believe that our tethered configuration approximates near-hovering conditions.

We collected data only from individuals that produced stable flight sequences and displayed the leg posture typical of free flight. Flies that exhibited short flight bouts and frequent landing responses after tethering were discarded. We generated both long- and short-exposure images of the particle flow in the fly's wake. Long exposures $(30 \mathrm{~ms})$ were used to visualize the gross structure of the wake over several wingbeat cycles. Shorter exposures $(0.5-1 \mathrm{~ms})$ were used to visualize flow dynamics within a single stroke cycle. The wingbeat analyzer generated a TTL pulse during the ventral flip of each wing stroke, which we used to trigger the CCD exposure. By varying the delay between the ventral flip pulse and the camera exposure, we could acquire images of the flow at different phases of the wingbeat cycle. Typically, four separate phaselocked images were superimposed on a single frame. This allowed us to examine a reasonable number of particle streaks without the necessity of bombarding the fly with an excessive density of Lycopodium spores for each exposure. These composite images, which were acquired over many different stroke cycles, could then be used to generate a phasereconstructed film of the wake dynamics within a single wingbeat. Quantitative analysis of the flow images was accomplished by measuring the length and direction of individual particle streaks and analyzing the resultant vector fields using the software program Transform (Spyglass).

\section{Force measurements}

The apparatus for measuring instantaneous forces is illustrated in Fig. 1D. The fly was tethered at the anterior notum to a $60 \mathrm{~mm}$ long, $0.1 \mathrm{~mm}$ diameter steel wire which was tuned to a frequency of $4.0 \mathrm{kHz}$ (with the attached fly). To measure the displacement of the wire along one of the main axes, a stationary shim was adjusted so as to leave a gap of $0.11 \mathrm{~mm}$ between its edge and the adjacent boundary of the wire. The wire's opposite boundary was concealed behind the edge of another shim (not shown in Fig. 1). A frequencymodulated beam from a diode laser $(670 \mathrm{~nm}, 2.5 \mathrm{~mW})$ was aimed through the gap to generate an interference pattern. The displacements of the wire relative to the fixed shim were transduced by a pair of photodiodes positioned $1.1 \mathrm{~m}$ away. We centered the two diodes on the intensity inflection points of the third minimum of the interference pattern, which was located approximately $20 \mathrm{~mm}$ from the central beam. The motion of the interference minimum enlarged the displacement of the wire by a factor of 180 and was transduced by the demodulated differential signal of the photodiodes. This differential signal could be calibrated as deflection force. Two sets of interferometry components (shims, laser, detector) were used in order to detect two orthogonal forces in the sagittal plane of the fly: $F_{\text {total-x }}$ (the forces parallel to the longitudinal body axis) and $F_{\text {total-z }}$ (the forces perpendicular to the longitudinal body axis). Oscillations of the wire were damped with short foam cylinders placed at either end of the wire. The wire ran through the center of each cylinder, and the outer surface of the foam cylinders was compressed by a rigid brass covering. Mechanically induced oscillations of the tethered fly on the wire were damped in the horizontal and vertical directions to less than $30 \%$ between subsequent peaks. The damped oscillation is expected to increase the actual time constant of the force measurement from approximately $40 \mu$ s (see below) to approximately $56 \mu \mathrm{s}$. However, the oscillation and the oscillation-induced increment of the time constant can be almost completely reduced by off-line conditioning of the force records with an appropriate zero phase-lag filter.

Reconstruction of lift and thrust forces from the morphologically defined vectors, $F_{\text {total-x }}$ and $F_{\text {total-z, depends }}$ upon the actual posture of a fly. A difference of $24^{\circ}$ between the elevation angle of the mean flight force and the body angle of Drosophila has been determined using a mechano-electrical transducer by varying the body angle during tethered flight in still air (Götz and Wandel, 1984). The angle between the mean force vector and the body does not vary with the absolute orientation of the body in space, at least for the investigated range between -45 and $+150^{\circ}$ with respect to the horizontal, and is unaffected by visual stimulation (Götz, 1968). An effect of airspeed on the angle between the mean force vector and the body axis is conceivable, but difficult to assess. During free flight, D. melanogaster is thought to select its airspeed by controlling the body angle in the range between approximately $60^{\circ}$ for hovering and approximately $30^{\circ}$ for cruising in a narrow box of $50 \mathrm{~cm}$ diameter (K. G. Götz, unpublished results). An almost linear relationship between body angle and airspeed has been found in a larger congeneric species, $D$. hydei: $60^{\circ}$ at $0 \mathrm{~cm} \mathrm{~s}^{-1}, 40^{\circ}$ at $40 \mathrm{~cm} \mathrm{~s}^{-1}$, and $20^{\circ}$ at $80 \mathrm{~cm} \mathrm{~s}^{-1}$ (David, 1978). During the fastest cruising flights in which the body axis is approximately aligned along the horizontal axis, $F_{\text {total-x }}$ would correspond to thrust and $F_{\text {total-z }}$ would correspond to lift. During hovering flight, when the animal maintains its body at an angle of about $60^{\circ}$ with respect to the horizontal, the thrust component becomes very small and the upward lift becomes the resultant of $F_{\text {total-x }}$ and $F_{\text {total-z. }}$.

\section{Interpretation of interferometric data}

The attempt to record small flight forces with a high temporal resolution confronted us with a series of challenging problems. We provide a short account of the physical assumptions underlying our interferometric methods to convey a feeling for the dimensions of the signals and the reliability 
of their measurement. The interferometer was calibrated, for each of the 16 flies, by repeated application of horizontal and vertical forces of $100 \mu \mathrm{N}$. However, time-variant flight forces resulting from a $190 \mathrm{~Hz}$ wingbeat are not necessarily transformed into equivalent deflections of the wire. These forces act against (i) inertial forces proportional to acceleration, (ii) frictional forces proportional to velocity and (iii) elastic forces proportional to the position of both the fly and the wire. A deflection of the taut wire by a force of $10 \mu \mathrm{N}$ (roughly the weight of a Drosophila) resulted in a displacement of $2.9 \mathrm{~nm}$, which corresponds to the length of a chain of 13 iron atoms in the wire. By using these values in an ordinary model of string mechanics, we estimated the time constant describing the transition to a new equilibrium to be $40 \mu \mathrm{s}$. Calculation of the actual time course of a deflection shows that the wire should approach asymptotically to within $1 \%$ of its equilibrium position in less than $1.7 \%$ of an average wingbeat period of $5.26 \mathrm{~ms}$. For this reason, the inertial forces resulting from the deflection of the combined wire and fly mass can be neglected. The same holds for the frictional forces. The frictional coefficient of a fly is approximately $4 \mathrm{mg} \mathrm{s}^{-1}$, estimated from the ratio of body weight to maximum falling velocity of an anesthetized fly with its wings spread. Assuming that this term dominates the drag of the fly-wire system, the resulting frictional force (the product of the frictional coefficient and velocity) is nearly five orders of magnitude smaller than the weight of the fly. These results allow us to derive the time course of the flight force directly from the deflection of the wire. In the absence of further complications, the time resolution should be limited primarily by the resonance frequency of the system.

However, two dynamic artifacts of the interferometer must be discussed in order to interpret the interferometric measurements properly. (i) We observed a permanent lowamplitude modulation of the force components in the region of $2.8 \mathrm{kHz}$ that was not correlated with the tension or deflection of the wire. This has tentatively been attributed to a vibration of the shims and was easily suppressed by averaging several wingbeat cycles. (ii) A transient resonance at approximately $1.8 \mathrm{kHz}$ was observed following deflection of the wire. This effect shows a high degree of damping and is inconspicuous in the absence of an attached fly. The effect was greatest when a flying or resting fly was mounted onto the wire and the wire was deflected at an oblique angle relative to the animal's body axis. The modulation frequency of this artifact did not increase significantly with the tension of the wire. These results suggest that this $1.8 \mathrm{kHz}$ phenomenon results from a damped oscillation of the tethered fly around the axis of the wire. We calculated the expected initial deflection due to a change in the flight force acting on the center of gravity, the resonance frequency of the excited oscillation and its repulsive action on the wire. Our estimates are comparable with the observed artifact. In principle, this artifact could be avoided (i) by shortening the distance between the center of gravity of the fly and its point of attachment to the wire, or (ii) by increasing the stiffness in torsion of the wire at the expense of sensitivity. So far, both approaches have proved technically impossible to implement, and the flight force recordings are potentially contaminated by this artifact. A conspicuous $1.8 \mathrm{kHz}$ oscillation did regularly appear in our recordings of the horizontal force component, $F_{\text {total-x }}$, during the upstroke (see Fig. 10A). This oscillation should be considered with caution, since it is likely to represent the reverberation of the fly's body mass around the wire rather than actual temporal fluctuations in flight forces. However, the $1.8 \mathrm{kHz}$ reverberation can be treated as a contribution from an independent oscillator that is linearly superimposed onto the recorded flight forces and whose time average is zero. Estimates derived from a mathematical model of the fly-wire system suggest that the time constant of our force measurements (approximately $56 \mu \mathrm{s}$ ) is not significantly lengthened by superposition of the comparatively slow oscillation. In some analyses, we attenuated the influence of the $1.8 \mathrm{kHz}$ oscillation by off-line conditioning with an appropriate zero-phase filter. This procedure removes the contribution of the contaminating reverberations, but diminishes the temporal resolution of our measurements by a factor of about two. This filtering is only justified if the force sensor is sufficiently linear so that its output remains balanced following superposition of large amplitude oscillations. In the present experiments, linearity up to at least $200 \mu \mathrm{N}$ was achieved by operating the wire at high tension so that its displacements were extremely small. In summary, we believe that the interferometer provides a sufficiently accurate and instantaneous record of flight forces at frequencies below $1.8 \mathrm{kHz}$.

\section{Results \\ Gross structure of wake}

Several important features of the wake structure are manifest in the long-exposure images. For reference, a cartoon reconstruction of the generation of a single vortex ring is shown in Fig. 8. The individual vortex loops generated during each wing stroke progress downwards, tracing the outer boundary of the wake which appears as an outer layer of vortical flow in the sagittal images of Fig. 2A-E. Inside the vortical layer, there is a central region of high-velocity linear flow that is formed from the central portions of the individual vortex rings. We term the structure traced out by the rearwardmoving vortex loops the vortex stack. As indicated in coronal sections (Figs 2F-J, 3), the cross section of the vortex stack near the fly is not circular, but rather has the outline of an inverted heart. The presence of a heart-shaped vortex stack is important because it provides evidence for the mechanisms of vortex shedding during the ventral reversal. As the wings rotate and change direction at the start of the upstroke, they rapidly shed vorticity along their lengths, leaving behind two vortex filaments that are continuous with the filaments shed during the downstroke (see Fig. 8). In many birds and insects that clap their wings at the bottom of the downstroke, the free distal ends of these vortex filaments fuse below the body to create a continuous circular vortex ring. The same is true for insects 


\section{M. H. Dickinson AND K. G. GöTZ}

Fig. 2. Long-exposure images indicate the boundaries of the vortex stack generated by a fly. (A-E) Sagittal sections through the wake of a fruit fly cut through the dorsal and ventral boundaries of an outer vortical layer produced as individual vortex rings translate backwards. Inside the vortical layer, the flow is linear as it moves through the central regions of the stacked vortex loops. The positions of the sections for each frame are indicated by the lines in Fig. 1C. In A, the top layer of vorticity originates from the tips of the wings in their dorsalmost position. The ventral boundary starts at the tip of the abdomen. The dorsal boundary $1 \mathrm{~mm}$ from the midline is similar (B), but the ventral boundary now extends forwards near the position of the wing during the ventral reversal. In $\mathrm{C}$, the section cuts a long tangent through the vortical layer with only a small portion that slices through the central region of the vortex stack. In D, the laser section just glances the lateral extent of the vortical layer. The section shown in E, $1 \mathrm{~mm}$ lateral to the wingbeat envelope, is outside the border of the vortical layer. $(F, G)$ Coronal sections of the wake seen from behind. Anterior to the head (F), there is no evidence of the fly's wake. The section through the wingbeat envelope near the wings' position at the time of the ventral flip $(\mathrm{G})$ shows the ventral boundary of the vortex stack. Note that the medial boundaries of the vortex stack are attached to the thorax of the fly. In more posterior sections $(\mathrm{H}, \mathrm{I})$, the ventral boundaries of the vortex stack move downwards, but are still attached medially to the body. In the most posterior section $(\mathrm{J})$, the dorsal boundary of the vortex stack is now visible, and the ventral attachment sites have slid off the tip of the abdomen. Scale bar, $1 \mathrm{~mm}$.

whose wings do not clap at the end of the downstroke (Grodnitsky and Morozov, 1992, 1993). Although the wings of Drosophila also reverse direction while still separated by a large ventral angle, the shed vortex filaments do not immediately fuse, but rather attach temporarily to the body. As the individual vortex loops move backwards, their ventral attachment points slide rearwards along the body towards the tip of the abdomen, where they finally fuse to complete a single continuous vortex loop. This fusion is required by Helmholtz's second law, which does not permit the infinite velocities that would exist were the free ends of the two vortex filaments to slip off the tip of the abdomen. Evidence for this attachment and subsequent fusion is seen in the coronal sections through the thorax (Figs 2F-J, 3), in which the attachment points may be traced posteriorly from the thorax to the tip of the abdomen. Sagittal images also provide evidence for the existence of vortex filament attachment to the body (Fig. 2A-E). Lateral sagittal sections slice through the top and bottom boundaries of the vortex stack, with a region of linear flow in between. However, no ventral vortex boundary is evident below the thorax in the midline sagittal view (Fig. 2A). Instead, the ventral boundary of the vortex stack originates at the tip of the abdomen, the location where the left and right filaments slide off the body and fuse, forming a continuous vortex ring. As the ventral attachment sites move rearwards, the vortex loop becomes distorted as visualized in the coronal flow sections (Figs 2F-J, 3). In coronal sections through the thorax, the vortex loops attach to the body at an angle of approximately $45^{\circ}$ with respect to the vertical (Figs 2, 3; frame G). However, as the loops pass backwards along the abdomen, this attachment angle grows more acute, so that as they pass along

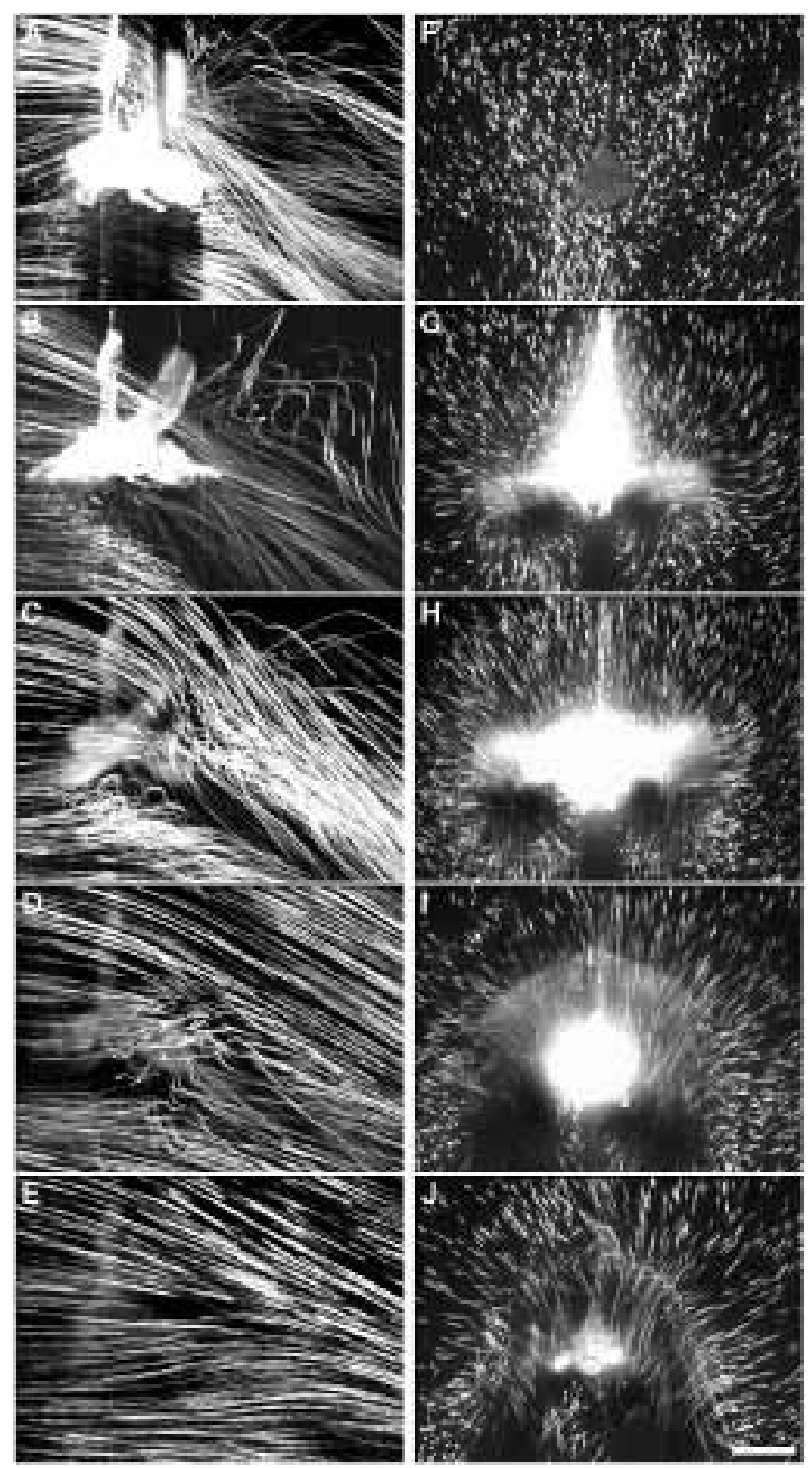

the tip of the abdomen the vortex layers on either side run nearly parallel to one another in a horizontal direction (Figs 2, 3; frame I).

\section{Dynamics of vortex ring formation}

After reconstructing the basic shape of the vortex rings from the long-exposure particle trajectories, we will now describe the formation of individual rings as visualized by shortexposure images of particle flow. All images were consistent with the formation of a single vortex ring made up of vorticity generated and shed during the downstroke and ventral flip. In this respect, the wake of a fruit fly is more similar to the general insect model described by Grodnitsky and Morozov (1992, 1993) than to the linked chain pattern described by Brodsky (1991, 1994). The clearest evidence for a single vortex ring is seen in phase-reconstructed films taken from a lateral sagittal plane, half the distance between the tip and base of the wing 

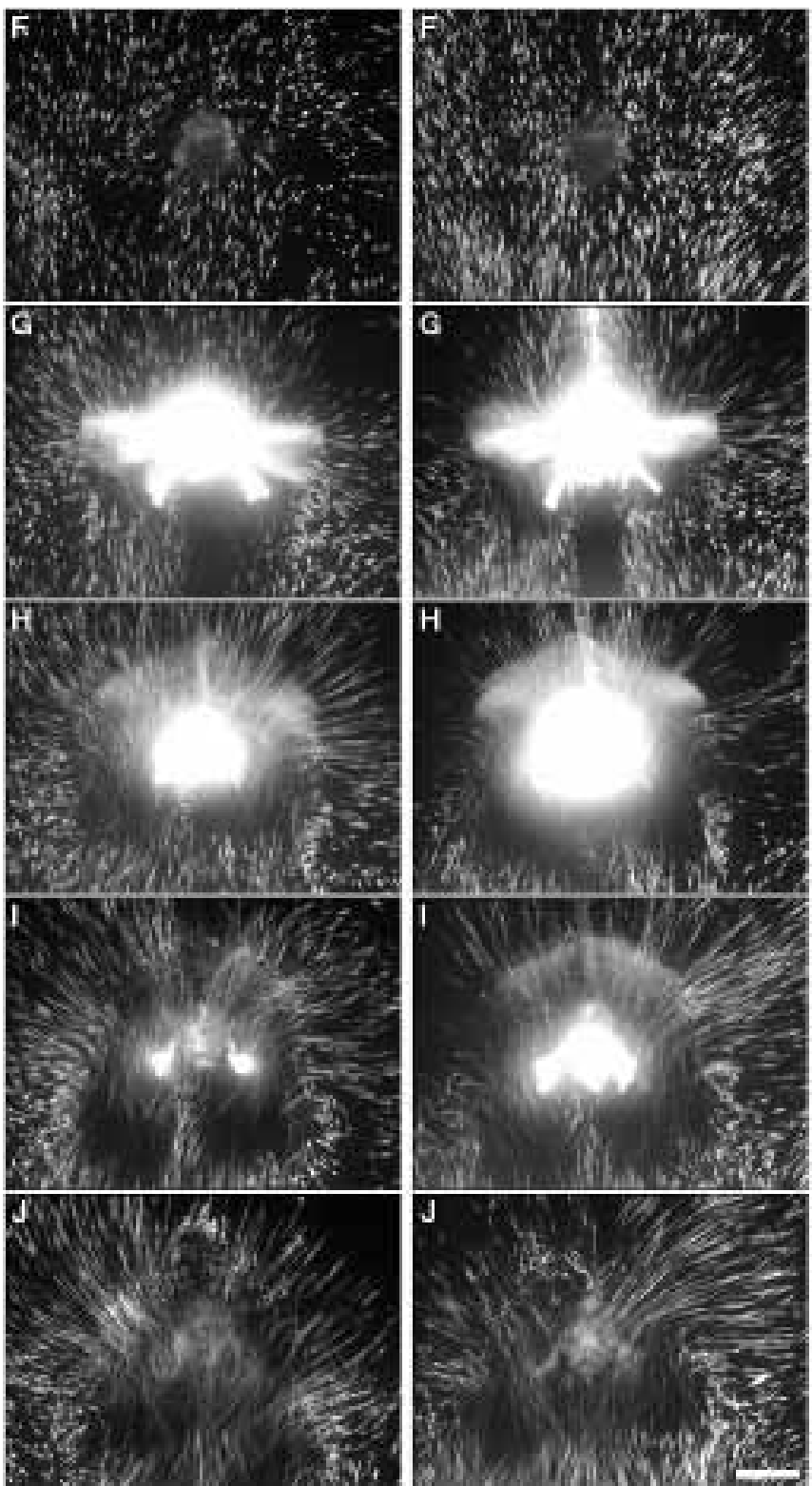

Fig. 3. The wake of a fruit fly has an inverted heart-shaped cross section. (F-J) Coronal sections similar to those in Fig. 2F-J are shown for two additional individuals in the left- and right-hand columns. The flies in these sections were aligned in a more dorsal position within the frame to better visualize the ventral portion of the wake. The corresponding sections between the two flies are not perfectly matched because of differences in the size of the individuals and laser alignment. Sections near the tip of the abdomen (I) show the inverted heart-shaped outline of the vortex stack that is caused by the medial attachment of vortex filaments. In sections posterior to the tip of the abdomen (J), the filaments have fused and the sharp ventral indentation in the vortex stack relaxes. Scale bar, $1 \mathrm{~mm}$.

(Fig. 1C, section M; Fig. 4). The flow moving rearwards from the stroke plane consists of long parallel particle streaks alternating with dark regions that are almost completely void of particles. These dark regions were very conspicuous features of our flow visualizations and could not be explained by shadows within the laser sheet or the inability to visualize rapidly moving particles. Whenever the fluid containing a uniform distribution of spores is accelerated, the particles should lag behind the surrounding air because of their greater density. Therefore, we interpret the dark areas as representing recently accelerated regions within the wake such as the highvelocity central portions of individual vortex loops. This interpretation is supported by the flow pattern at the dorsal and ventral boundaries of the dark segments in Fig. 4 (arrow in frame d5). The air flow constricts while passing through the dark regions, which is to be expected if the dark regions lie close to the plane of the vortex ring. Further, the dark regions originate behind the trailing edge of the wing during the downstroke and enlarge during the ventral flip (Fig. 4, frames $\mathrm{d} 4-\mathrm{u} 1)$. No comparable dark, high-velocity region is generated during the upstroke (Fig. 4, frames u1-u3), although the particle visualization may not be sensitive enough to detect weak vorticity. We conclude that one vortex loop is created during each stroke and that it consists primarily of the tip vorticity shed gradually during the downstroke together with the vorticity shed rapidly along the lengths of the wings during the ventral flip. An upstroke vortex loop, if present, is certainly much weaker than the downstroke loop and could not be unambiguously resolved by our methods. Fig. 5 shows a cartoon tracing of this interpreted pattern of vortex loop formation from a selection of frames in Fig. 4.

The rapidity with which vorticity is shed during the ventral flip is most clearly seen in phase-reconstructed images of particle trajectories in a coronal plane through the anterior portion of the thorax (Fig. 6). As the wings rapidly supinate (Fig. 6, frames d5-u1), regions void of particles develop below the wings, bordered ventrally by parallel arrays of long downwardly directed particle streaks. These dark particle voids, now viewed from the rear, are the same as those seen moving backwards in the sagittal plane visualizations (Fig. 4) and indicate the high-velocity flow through the center of the vortex loops, now oriented outwards from the plane of the image. Following the ventral flip, the dark sections representing central portions of the vortex rings move rearwards out of the plane of section (Fig. 6, frames u2-dr). During most of the wing stroke, there is no indication of vorticity anterior to the wing hinge. Given the dramatic accelerations of the fluid during the ventral flip, it would appear that the strength of the vorticity shed from the wings during this rotation is greater than the tip vorticity shed during the downstroke. It is possible, however, that this impression is an artifact caused by the plane of section.

Fruit flies and other small insects are known to display 'squeeze, clap and fling' kinematics during the dorsal stroke reversal (Weis-Fogh, 1973; Ellington, 1984b; Götz, 1987). The flows generated by this behavior influence the wake structure by modulating the fluid velocity above the body in the central portion of each vortex loop (Fig. 7). The resolution of our images is not precise enough to determine the exact changes in flow induced by the dorsal closure of the wings, but the squeeze and clap kinematics appear to have important 


\section{M. H. Dickinson AND K. G. GöTZ}

Fig. 4. Phase-reconstructed images of wake dynamics visualized from the mid-wing sagittal plane (position $\mathrm{M}$ in Fig. 1C; see also nearby sections $B$ and $C$ in Fig. 2). The images were taken at 10 equally spaced points within the wingbeat cycle. The animal was flying at a wingbeat frequency of approximately $200 \mathrm{~Hz}$, so the temporal interval between images is roughly $500 \mu \mathrm{s}$. In order to increase the spatial sampling of the images, each frame shows a multiple exposure of four separate images at the same phase point. In this and several subsequent figures (Figs 5-8), the frames are labeled according to their approximate position within the wingstroke: vf, ventral flip; u1, $\mathrm{u} 2$ and $\mathrm{u} 3$, three sequential positions in the upstroke; dr, dorsal reversal; d1, d2, d3, d4 and d5, five sequential positions within the downstroke. The sequence begins at the start of the downstroke (d1-d3), when the fly begins to make a new vortex ring as the wings shed vorticity. By late downstroke $(\mathrm{d} 4, \mathrm{~d} 5)$, the central region of the vortex ring is clearly visible as a crescent-shaped dark region just behind the wing. Notice the indentation of the flow at the dorsal boundaries of the vortex ring (arrow). At the time of the ventral flip (vf), the wing rapidly sheds its bound vorticity which appears as the small dark region directly below the wing. During the upstroke (u1-u3), the shed vortex ring moves backwards, and the motion of the wing does not appear to generate vorticity. By the time of the clap and fling (dr) (not visible in this section), the vortex ring of the previous downstroke has moved beyond the posterior tip of the abdomen. Scale bar, $1 \mathrm{~mm}$.

consequences for the periodicity of fluid velocity over the body. First, during the upstroke, the velocity of air over the dorsal surface of the body appears to increase as the area between the wings rapidly decreases (Fig. 7, frames vf-u2). This effect is probably due simply to the law of continuity increased fluid velocity in a smaller cross-sectional area. Second, just prior to the clap, the close proximity of the two leading edges of the wings presents an instantaneous barrier to flow over the sagittal midline (Fig. 7, frame u3). This transition in flow from an initial increase to an eventual decrease in speed as the wings draw closer together is reminiscent of the 'paddle or rake' mechanism described by Cheer and Koehl (1987) for copepod feeding. Finally, during the clap, the closure of the trailing edges of the wings squeezes fluid rearwards, increasing the velocity of air over the thorax and abdomen (Fig. 7, frames $\mathrm{dr}, \mathrm{d} 1$ ). Taken together, the dorsal stroke reversal appears to generate a more pulsatile flow over the body.

The flow visualizations indicate several important differences between the wake of a tethered fruit fly and the simple schematic wakes proposed by vortex models of flight (Rayner, 1979b; Ellington, 1984c). Within a simple circular vortex ring, there is one central region through which the linear velocity of the fluid is high. Inside the heart-shaped vortex loops of a fruit fly, the velocity distribution is more complex because the central region may be roughly subdivided into three flow corridors (Fig. 8). The midline dorsal corridor originates above the body and follows the outline traced by the wing tips. The velocity of air flow within this corridor is probably increased by the squeeze and clap kinematics of the wings. The large ventral angle between the wings during the ventral flip ensures that the filaments of shed wing vorticity

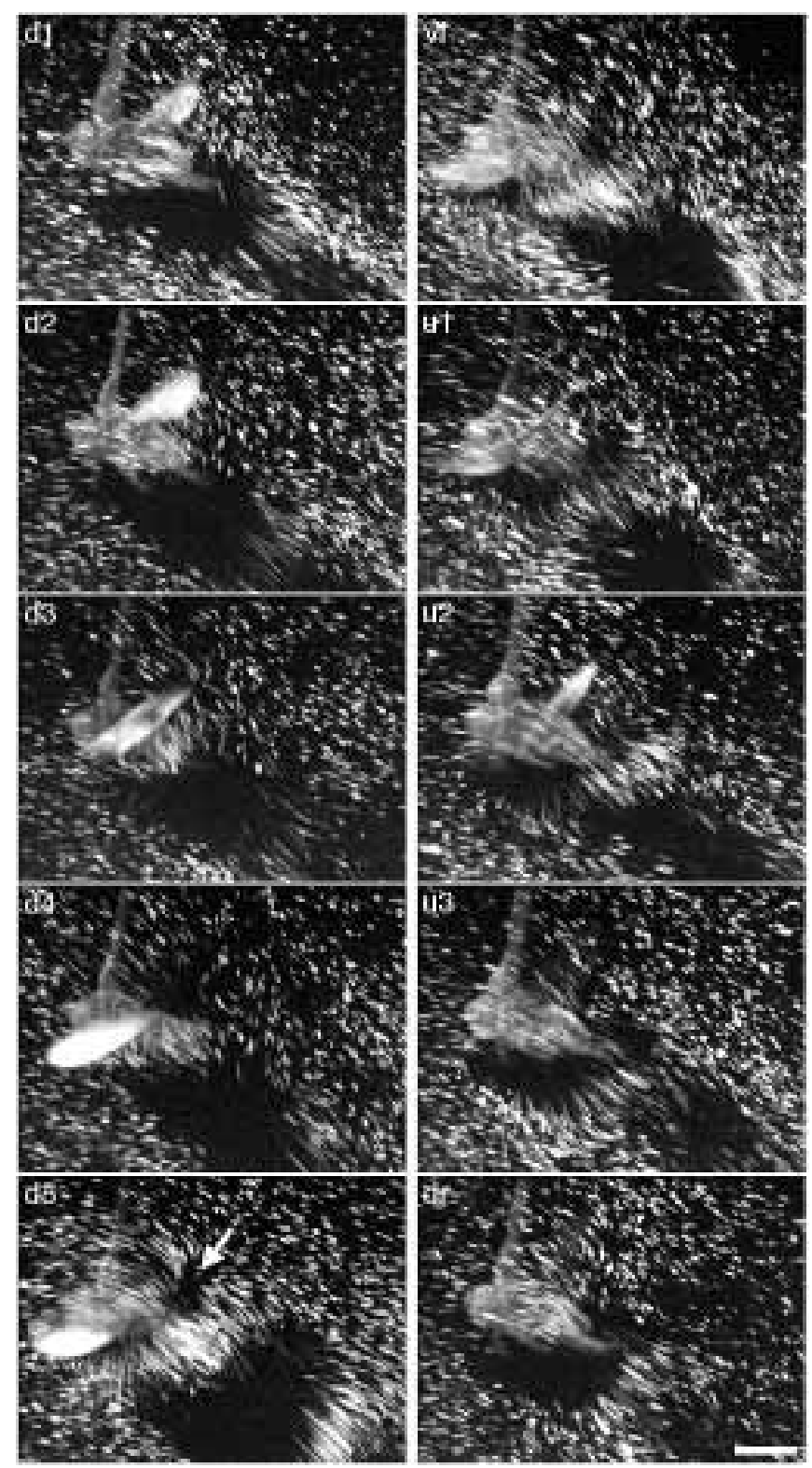

will not fuse but rather attach to the body, enclosing the two ventral corridors of high-velocity flow bounded medially by the body. The existence of the three flow corridors immediately behind the stroke plane has been verified by Lehmann (1994) in a three-dimensional reconstruction of a fruit fly's wake using hot-wire anemometry. This complex structure of the wake may be related to the flight control capabilities of the animal, since the presence of two lateral flow corridors indicates that the force-generating capabilities of the two wings are at least partially independent. Indeed, in the study mentioned above, Lehmann found that the relative velocity in the two lateral corridors changes during visually induced turns or when a specific steering muscle (the second basalar) was stimulated. His measurements also indicated that the three corridors eventually coalesce into a single circular section $5 \mathrm{~mm}$ posterior to the stroke plane. Quantitative measurements of the flow velocity within the wake are shown in Fig. 9, in which 
Fig. 5. Cartoon reconstruction of several frames (d2, d5, u1 and dr) in the the flow visualization of Fig. 4. Dorsal and ventral sections through the developing vortex ring are indicated by circles, with arrows marking the orientation of vorticity. See the legend to Fig. 4 for more details.

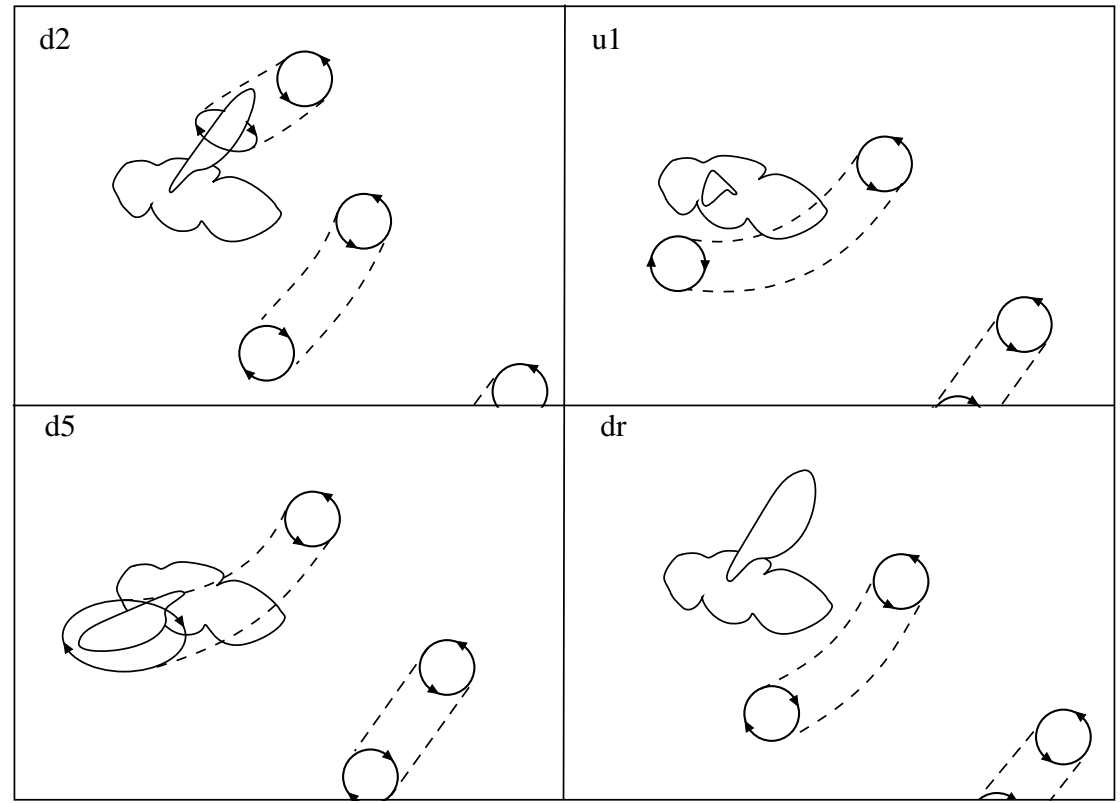

the streak lengths of the images in Figs 4 and 7 have been used to construct a map of average fluid velocity and flow streamlines in two sagittal planes. The central axis of the wake is oriented at approximately $25^{\circ}$ with respect to the longitudinal body axis, which is consistent with the $24^{\circ}$ elevation of the mean flight force vector measured by Götz and Wandel (1984). The velocity gradient across the body over the sagittal midline is quite large, and the resultant pressure differential could potentially act as a source of body lift (Fig. 9A,B). However, using our flow measurements, we estimate the pressure differential across the body to be approximately $0.07 \mathrm{~N} \mathrm{~m}^{-2}$, which would produce a body lift of only $0.13 \mu \mathrm{N}$ or approximately $1 \%$ of the total body weight. This is a very rough estimate, however, based on a time average of the flows and must be considered with caution. The velocity map in a more lateral sagittal plane (Fig. 9D) shows a longitudinal section through one of the ventral corridors. The peak flow velocity appears lower than that over the sagittal midline, but this is probably an artifact due to the inertia of the visualization particles within the highly time-variant flow of the lateral corridors. The Lycopodium spores should not accelerate to the peak air velocity because of their greater density. In addition, the inertia of the particles may make them accumulate in the outer vortical layer. The resultant paucity of particles within the dark regions decreases the accuracy of the interpolation algorithm used to reconstruct the velocity flow field.

\section{Measurement of total flight forces}

Using laser interferometry, we were able to measure the instantaneous net flight forces produced by tethered flying Drosophila (Fig. 10). In order to construct both the magnitude and the orientation of the net force vector generated by the flies, we simultaneously recorded the force components parallel and perpendicular to the longitudinal body axis
( $F_{\text {total-x }}, F_{\text {total-z }}$, respectively). To improve the signal-to-noise ratio and to sample over an extended time period, we superimposed an average of 987 sweeps of every nineteenth stroke cycle in each of 221 flight sequences from 16 flies. The signal-averaging was possible by triggering on the ventral flip timing pulse that was generated by the wingbeat processor. In total, the results represent approximately $6 \mathrm{~h}$ of flight on the interferometer. The timing pulse was also used to trigger a stroboscope after a variable delay. This allowed us to inspect the kinematics of the dorsal reversal and to measure its timing (Götz, 1987). To ensure that the averaging procedure did not obscure temporal relationships, we limited our quantitative analysis to long stable flight sequences with less than $3.5 \%$ variation in wingbeat frequency. The average wingbeat period of the sequences that met this criterion was $5.26 \pm 0.26 \mathrm{~ms}$ (S.D.). In order to average separate flight sequences together, we normalized the time base of each record to the wingbeat period.

We attempted to measure the magnitude of the mean flight force by abruptly terminating each flight sequence using a fine probe to obstruct the wing path. However, this procedure resulted in a force offset that was of comparable magnitude to the mean flight force. The offset arose because the mechanical force required to stop the fly (which was much greater than the peak magnitude of the flight force) caused the ends of the wire to catch on the damping foam. However, the relaxation time of the unwanted offset was larger than the cycle time by at least three orders of magnitude. This allowed us to separate, by computer-aided subtraction of the mean values of $F_{\text {total-x }}$ and $F_{\text {total-z, }}$, reliable measurements of the fluctuation of these forces within a stroke cycle from less reliable measurements of their means. Without the necessity for excessive temporal resolution, the mean forces can be most accurately determined using the mechano-electrical transducer mentioned in an earlier section (described in Götz and Wandel, 1984). The 

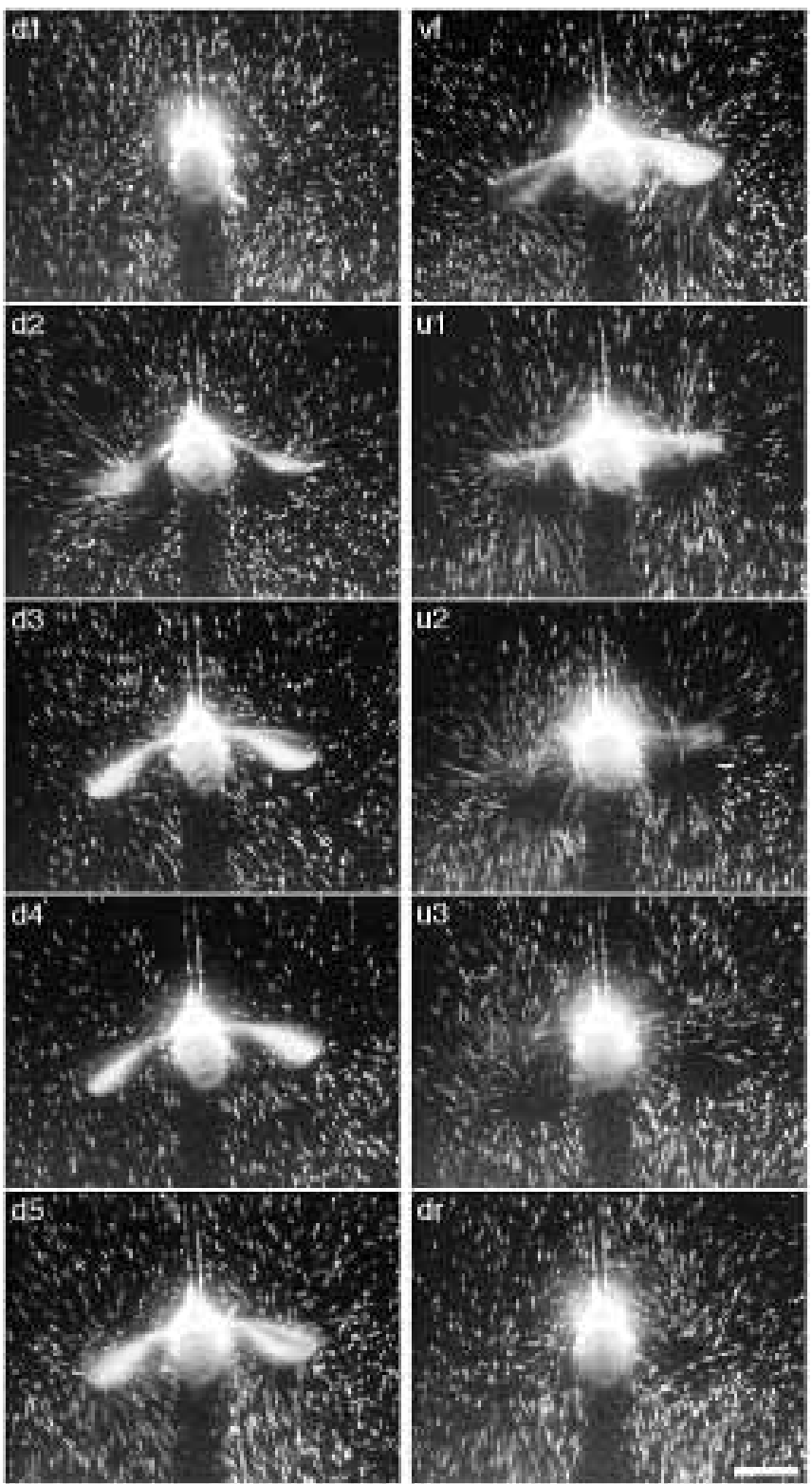

Fig. 6. Phase-reconstructed images visualized in coronal sections through the wingbeat envelope near the position of the wings at the time of the ventral flip (position $\mathrm{G}$ in Fig. 1C; see also the corresponding section in Fig. 2). The sequence of images is roughly equivalent to those shown in Fig. 4. The wake is viewed from the rear through a window in the flow tunnel. During the downstroke (d1-d5), the wings move forwards but do not greatly alter the flow within the plane of visualization. During the ventral flip (vf), the fluid accelerates as vorticity is shed along the wings. Notice that, in this particular image, the left wing rotates slightly in advance of the right wing. Just after the ventral flip (u1), the wings have supinated and begun to move rearwards out of the plane of section. The ventral boundary of the vortex stack is now visible just below the two dark regions under each wing. The lateral boundaries of the vortex stack are evident in the rotational flow present at the tips of the wings. During the upstroke and dorsal reversal ( $\mathrm{u} 2-\mathrm{dr}$ ), the wings move out of the plane of visualization, as do the dark regions representing the two lateral corridors of high-velocity fluid within the vortex stack. Scale bar, $1 \mathrm{~mm}$.
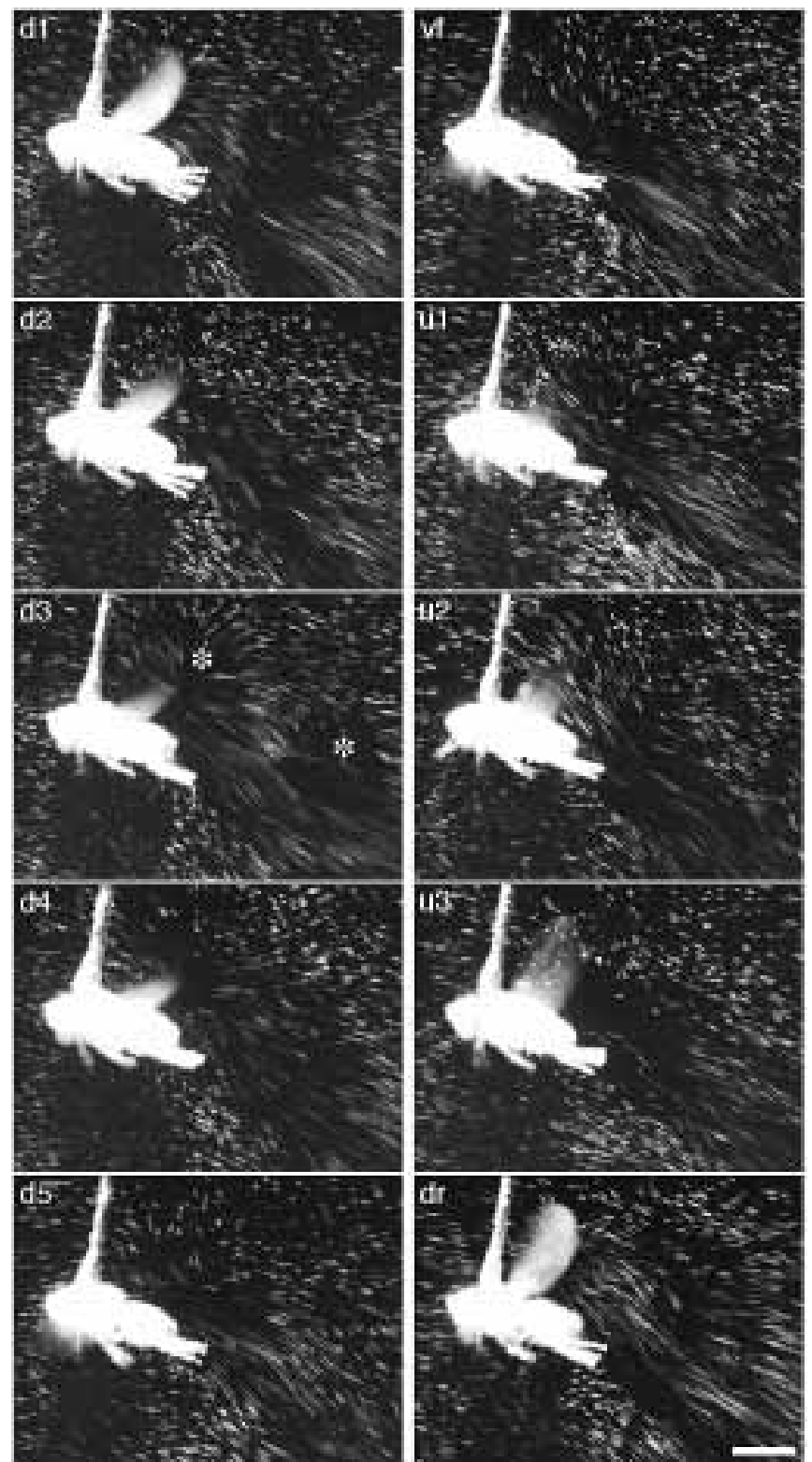

Fig. 7. Phase-reconstructed images of the flow visualized in the midsagittal plane illustrating the squeeze, clap and fling behavior (position A in Fig. 1C; see also the corresponding section in Figs 2 and 3). Again, the sequence of images is approximately equivalent to those shown in Figs 4 and 6 . Note that in all sections there is a large velocity gradient across the dorso-ventral axis of the fly. After the squeeze (d1), the leading edges of the wing are first to leave the plane of visualization as the wings peel apart. A new vortex loop develops as the wings move apart (d2). In frame d3, dark regions at the positions of the developing and recently shed vortex loops are especially clear (asterisks). At mid downstroke (d4), the wing has moved out of the plane of visualization. From the end of the downstroke to the start of the upstroke (d5-u2), the vortex loop moves backwards at a velocity of approximately $0.4 \mathrm{~m} \mathrm{~s}^{-1}$ as indicated by the dark region above the body. During the clap (u3, dr), the wings are clearly visible above the dorsal midline. The fluid velocity between and behind the wings becomes small, presumably as a result of the obstruction caused by the close apposition of the leading edges. The fluid velocity behind the wings greatly increases as the trailing edges squeeze together $(\mathrm{dr})$. Scale bar, $1 \mathrm{~mm}$. 
d1

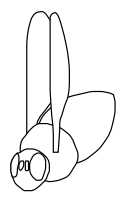

d2

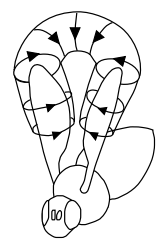

d3

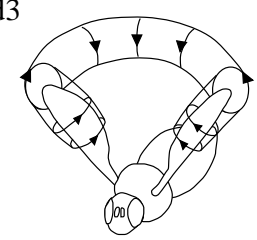

d4

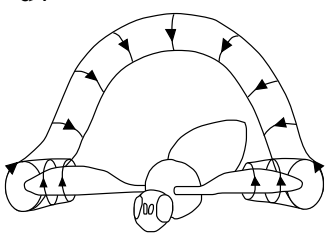

d5

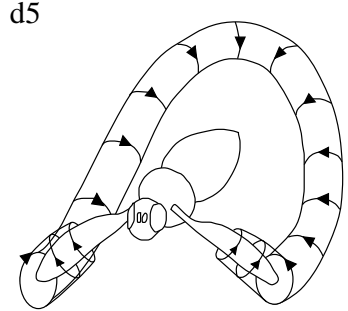

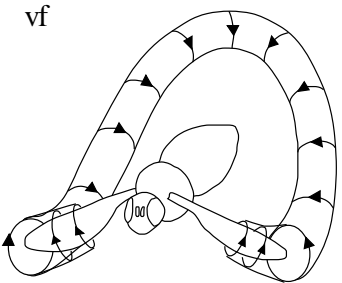

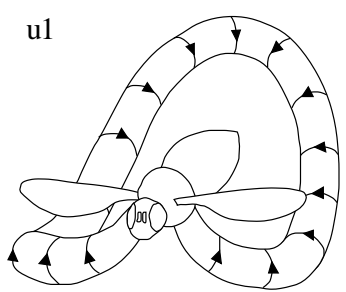

u2

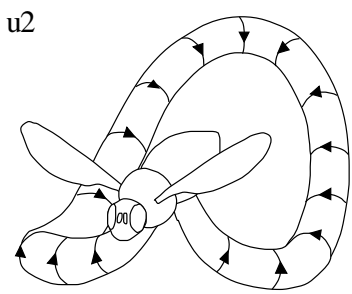

u3

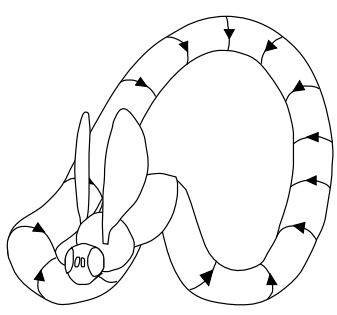

$\mathrm{dr}$

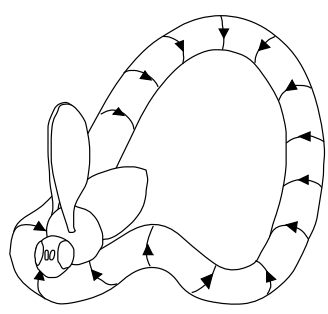

Fig. 8. Cartoon reconstruction of vortex loop formation by Drosophila (d1-d5). The vortex loop forms as the wings move apart during the downstroke. During the ventral flip (vf), the bound vorticity of both wings is rapidly shed. During the upstroke (u1-u3), the wings generate no additional vorticity and the ventral ends of the vortex filaments stay attached to the body as the loop moves backwards. During dorsal reversal (dr), the trailing edges of the wings squeeze together. By this time, the vortex loop has closed as the two ends detach from the tip of the abdomen and fuse.

magnitude of the mean force vector, recorded under the conditions of both the flow visualization and the interferometer experiments, was $10.8 \pm 0.5 \mu \mathrm{N}$ (S.E.M., 74 sequences from 17 flies) in still air and $10.4 \pm 0.4 \mu \mathrm{N}$ (S.E.M., 90 sequences from 19

flies) in a $20 \mathrm{~cm} \mathrm{~s}^{-1}$ air flow. Since the forces measured in still and moving air were not significantly different (Fig. 10A), we pooled the data, yielding a total mean of $10.6 \pm 0.3 \mu \mathrm{N}$. This force is the equivalent of the average body weight of a female Drosophila. As mentioned above, the elevation angle of this vector in the $x-z$ plane is approximately $24^{\circ}$. The corresponding components of the mean flight force amount to $9.7 \mu \mathrm{N}$ for $F_{\text {total-x }}$ and to $4.3 \mu \mathrm{N}$ for $F_{\text {total-z. In the following }}$ analyses, these values replace the less reliable mean flight forces obtained using the interferometer. All of the 16 flies were tested alternately in still air and in the presence of a $20 \mathrm{~cm} \mathrm{~s}^{-1}$ air flow identical to that used in the flow visualization experiments. As shown in Fig. 10A, the differences in the time course and magnitude of the force measurements under these two conditions were small compared with the variation among individuals. The similarity of the force records holds for each of the 16 flies and is not unexpected considering that a background air flow of $20 \mathrm{~cm} \mathrm{~s}^{-1}$ produces an effective advance ratio of only 0.085 . The close coincidence of the two independent data sets throughout the stroke cycle demonstrates the rigorous reproducibility of the interferometric force measurements and allowed us to pool the results for further evaluation. The magnitude and time history of forces measured in these experiments were consistent across averaged sequences. The limiting curves in Fig. 10A represent the standard deviation (S.D.) of the pooled data from 221 flight sequences. The corresponding standard errors (7\% of the S.D.) and the $95 \%$ confidence limits ( $13 \%$ of the S.D.) of the means are too small to be represented graphically.

As seen in the $F_{\text {total-x }}$ waveform in Fig. $10 \mathrm{~A}$, the forces developed during the ventral flip appear transiently to drive the fly on the wire to a resonance of about $1.8 \mathrm{kHz}$, which is a factor of 0.45 lower than the resonance frequency of the tuned wire with an attached fly. Tapping the wire elicits a similar oscillation, regardless of whether the animal is flying. For this reason, these oscillations do not seem to be attributable to flight-associated periodic processes such as rapidly changing aerodynamic forces or inertial forces resulting from rotation or deformation of the wing during the ventral flip. In an earlier section, we described a resonance of similar frequency that appears to result from a twist-induced oscillation of the tethered fly about the axis of the taut wire. One possibility, therefore, is that the change in flight force at the start of the upstroke elicits a $1.8 \mathrm{kHz}$ oscillation of the body mass around the wire. Alternatively, the oscillations might be induced, not by the flight forces per se, but rather by the changes in angular momentum resulting from wing rotation during the ventral flip. The extreme angular acceleration and deceleration during the ventral flip is likely to exceed $\pm 3 \times 10^{8}$ degrees $^{-2}$ (see Fig. $6 \mathrm{~b}$ in Zanker and Götz, 1990). Conservation of the angular momentum requires the induction of torque pulses of up to $4 \mathrm{nN} \mathrm{m}$ about an axis parallel to the wire. The time course and magnitude of either these pulses or the compensatory forces (which may exceed $5 \mu \mathrm{N}$ ) appear to be sufficient to induce the observed oscillatory reaction of the wire. Whether elicited by flight forces or rotational momentum, the $1.8 \mathrm{kHz}$ oscillations 

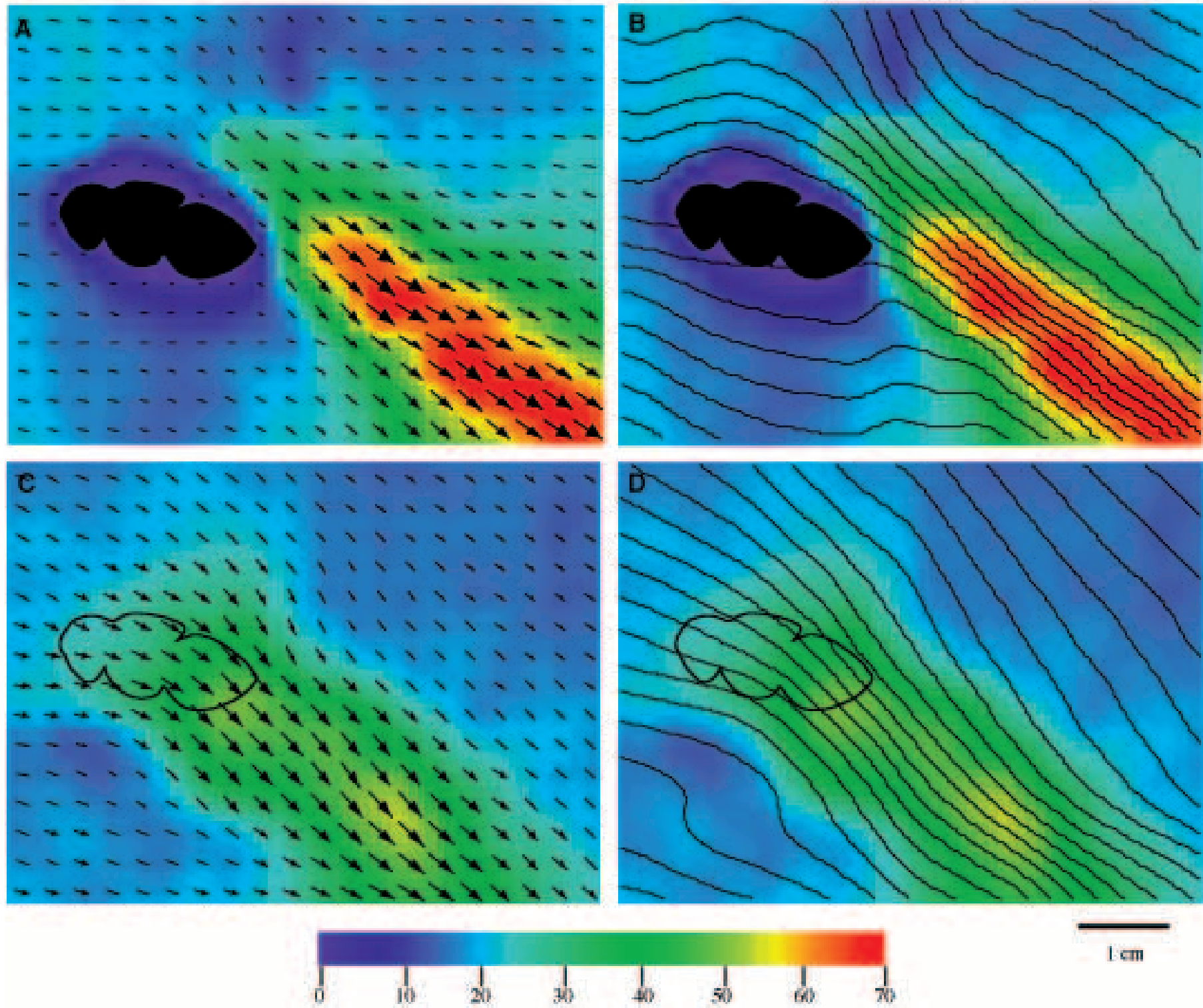

$1 \mathrm{~cm}$

Air velocity $\left(\mathrm{cm} \mathrm{s}^{-1}\right)$

Fig. 9. Spatial map of time-averaged fluid velocity of the wake indicating the midline (A,B) and ventro-lateral (C,D) flow corridors. The data in $\mathrm{A}$ and $\mathrm{B}$ were determined by analyzing the particle trajectories in Fig. 7. The data in $\mathrm{C}$ and $\mathrm{D}$ were determined by analyzing the images in Fig. 4. In all panels, the fluid velocity is encoded by pseudo-color according to the scale at the bottom. As explained in the text, the air velocity is probably underestimated in the ventro-lateral corridors. The frames on the left indicate the orientation of fluid velocity, and the right-hand panels show the streamlines of the flow calculated from the spatial gradient of the velocity field.

are not likely to represent the time course of changing aerodynamic forces and have been filtered in the reconstructions presented in Fig. 11.

The time courses of the total flight force records in Fig. 10A are qualitatively similar to those recorded by G. Nalbach (resonance frequency $2 \mathrm{kHz}$; unpublished results) for Calliphora erythrocephalia, but quite different from those published for Sarcophaga bullata (Buckholz, 1981). Buckholz attached his flies to a copper wire of low tensile strength and recorded the orthogonal components of a just-visible vibration by measuring reflected light. From the data presented in his paper, we estimate that the time constant for the asymptotic transition to a new equilibrium is at least two orders of magnitude larger than that in the present experiments. The resulting effects of inertia and friction complicate the interpretation of his data and may explain the large discrepancy between the results.

\section{Reconstruction of aerodynamic forces}

The raw signals transduced by the sensor attached to a fly's thorax represent the sum of aerodynamic and inertial forces ( $F_{\text {total-x }}$ and $\left.F_{\text {total-z }}\right)$. The inertial forces $\left(F_{\text {inert-x }}\right.$, and $\left.F_{\text {inert-z }}\right)$ 
result when the mass of the animal's wings and the added mass of the surrounding air are accelerated during the wing stroke. While the inertial forces averaged over an entire wing stroke are zero, they are transiently quite large and must be subtracted from the total measured forces in order to reconstruct the instantaneous aerodynamic forces $\left(F_{\text {aero-x }}\right.$ and $\left.F_{\text {aero-z}}\right)$. Zanker and Götz (1990) have previously calculated the inertial forces using the data of Zanker (1990a), which were based on reconstructed kinematics of 25 phaseequidistant points within the wing stroke. We interpolated these calculated waveforms to match our sampling rate (100 points per wingstroke) by using a cubic spline function (Fig. 10B). Before subtraction, the two data sets were aligned, matching the extreme downstroke position in Zanker's data with the timing of the ventral flip pulse in the force recordings. However, it should be noted that the accuracy of the temporal alignment is limited by the $200 \mu$ s effective sampling interval of the kinemtic data. In addition, the reconstruction of aerodynamic forces by inertial subtraction requires several simplifying assumptions. (i) The inertial forces were calculated from averaged wing-stroke kinematics. This may have a smoothing effect on the time course. Note, however, that the same holds true for the averaged time course of the recorded flight forces. (ii) Inertial force components due to pronation or supination of the wings were neglected. This is justified only in the case of rigid wings and a sufficiently symmetrical distribution of the total wing mass around the axis of rotation. (iii) The inertia resulting from the mass of the wing and the virtual mass of the bound air cannot be determined with sufficient accuracy. As a small fraction of the resulting mass, the virtual mass has been replaced in the calculation by a point mass near its estimated center of gravity on the wing. In order to assess the inaccuracies that might be introduced by these assumptions, we varied the magnitude of the inertial force trajectories used in the reconstructions of aerodynamic forces. As indicated in Fig. 10C, the calculated aerodynamic forces are qualitatively similar when using inertial force magnitudes of $75 \%, 100 \%$ and $125 \%$ of those calculated by Zanker and Götz (1990). Thus, although the unknown contribution of rotational inertia remains a potential source of error, it is unlikely that our reconstructions would be compromised by serious inaccuracies in the estimation of the magnitude of translational inertia. According to earlier estimates (Zanker and Götz, 1990), the time course of the total flight force is expected to resemble the time course of its dominant component, the inertial force. Comparison of the corresponding curves in Fig. 10B illustrates the expected similarity. Evaluation of the actual data corroborates this impression: the correlation cannot be significantly improved by varying the magnitude and phase of the curves calculated by Zanker and Götz (1990) for the inertial forces. It is not easy to explain this coincidence if the corresponding forces were not measured or calculated with sufficient accuracy in their magnitude and phase.

The orthogonal aerodynamic forces were used to reconstruct trigonometrically the time history of the direction and magnitude of the total aerodynamic force generated by a flying Drosophila (Fig. 11A). Because high frequencies within the signals do not represent components of the flight forces, we have also plotted low-pass-filtered versions of the data records. The magnitude of aerodynamic forces within each wing stroke oscillates between segments of high and low force generation. The total flight force rises to a plateau of approximately $35 \mu \mathrm{N}$ during the downstroke and then falls during the second half of the upstroke. There appears to be a transient peak in the unfiltered version of the reconstructed flight force during the upstroke which exceeds $80 \mu \mathrm{N}$ although, as discussed previously, this is probably an artifact due to the $1.8 \mathrm{kHz}$ oscillation around the axis of the wire. The fluctuations in force magnitude are in phase with large changes in the orientation of the force vector. The largest flight forces are oriented roughly forward along the body axis. In contrast, the lowmagnitude flight forces during the late upstroke and early downstroke are oriented upward and rearward.

The body of a free-flying Drosophila moving at $20 \mathrm{~cm} \mathrm{~s}^{-1}$ should be inclined at an angle of approximately $50^{\circ}$ with respect to the horizontal (David, 1978). In Fig. 11B, we have reconstructed the time histories of the lift and thrust vectors under these free-flight conditions on the basis of our interferometric measurements. Again, we show both filtered and unfiltered versions of the reconstructed waveforms. With the body inclined at $50^{\circ}$, the wings generate upward lift from the middle of the downstroke to the end of the upstroke. Lift then declines to small negative values during the start of the downstroke. Thrust, by contrast, oscillates between nearly equal positive and negative extremes. Thrust is negative (rearward) from the end of the upstroke to the middle of the downstroke, at which time it rises quickly (within $0.9 \mathrm{~ms}$ ) to a peak positive value of approximately $30 \mu \mathrm{N}$. Thrust subsequently declines during the upstroke to reach a peak negative value by the start of the downstroke. The mean lift and thrust values at an inclination of $50^{\circ}$ are 10.2 and $3.0 \mu \mathrm{N}$ respectively.

On the basis of the flow visualizations, the largest flight forces are expected to occur during the downstroke and ventral flip when the wings appear to generate the most circulation (Fig. 6). However, from the traces in Fig. 11, it is clear that measured forces are greatest from the middle of the downstroke to the end of the upstroke. Thus, the measured flight forces are delayed by about $1 \mathrm{~ms}$ with respect to our expectations based on the flow visualizations.

\section{Discussion}

Any coherent theory of animal flight must be able to integrate the dynamics of wake formation with the dynamics of force generation. In this study, we have attempted to correlate the instantaneous aerodynamic forces produced by a fly with the time history and structure of its vortex wake. In each wing stroke, the fly produces a single heart-shaped vortex loop that is composed of vorticity generated and shed during the downstroke and ventral reversal. The wings appear to shed 

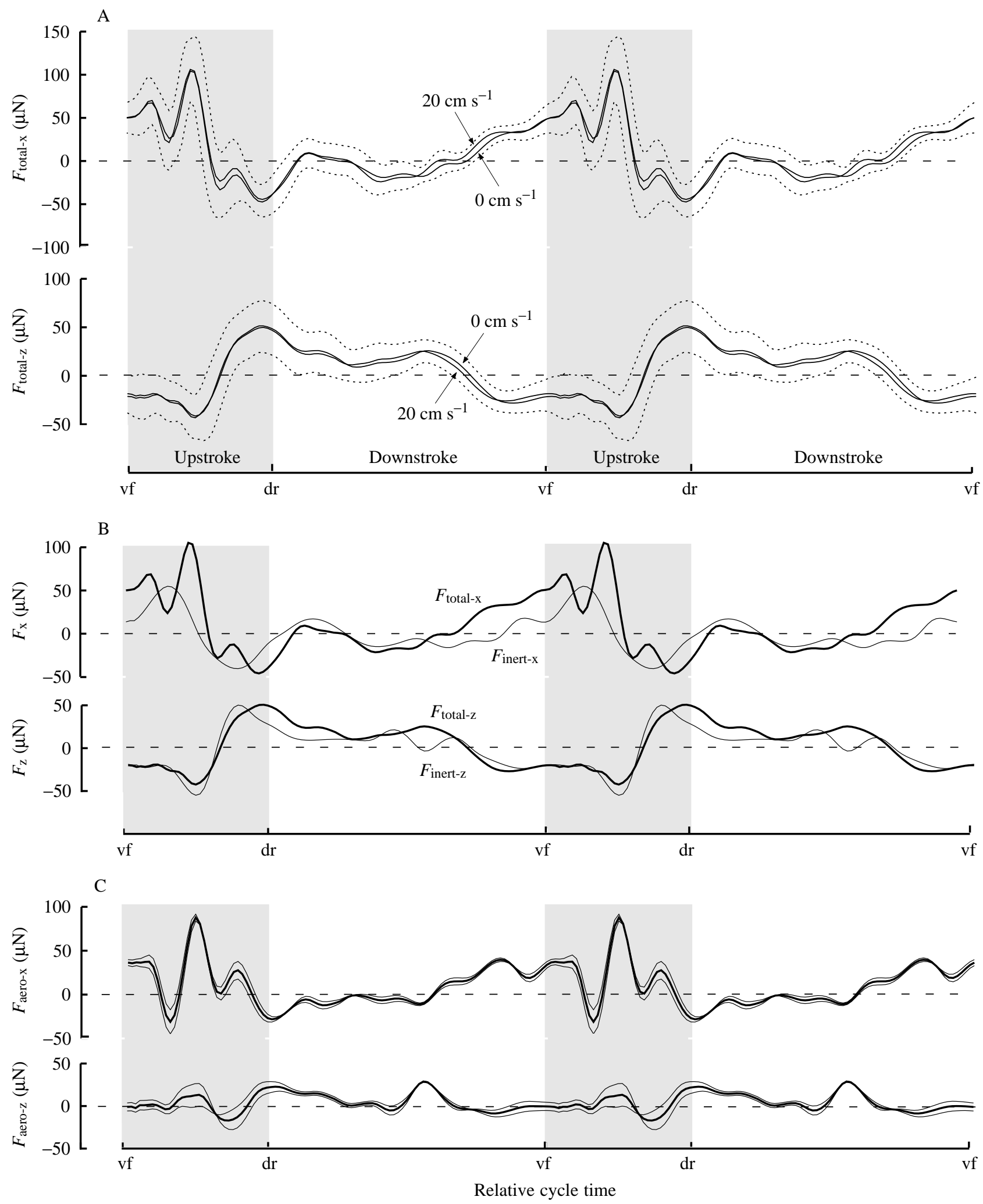

Fig. 10

little or no vorticity during the upstroke. In this way, the wake structure is similar to that previously reported for birds (Kokshaysky, 1979; Spedding et al. 1984; Spedding, 1986) and some insects, including the house fly Musca domestica (Grodnitsky and Morozov, 1992, 1993). However, the wake of a fruit fly differs from all previous descriptions of single-loop 
Fig. 10. The time history of flight forces within the wingstroke. (A) Total instantaneous forces parallel $\left(F_{\text {total-x }}\right)$ and perpendicular $\left(F_{\text {total-z }}\right)$ to the longitudinal body axis of the fly. Averages are shown from 16 individual flies at an airspeed of $0 \mathrm{~cm} \mathrm{~s}^{-1}$ (111 flight sequences) and $20 \mathrm{~cm} \mathrm{~s}^{-1}$ (110 flight sequences). The standard deviations of the pooled data are indicated by the dotted lines (the standard deviations of the two groups coincide almost exactly with these lines). Throughout this figure and Fig. 11, the data are plotted over two normalized stroke cycles to emphasize the periodicity of the flight forces. (B) The averaged total flight forces, $F_{\text {total-x }}$ and $F_{\text {total-z }}$ (221 sequences, thick lines), are shown superimposed with calculated inertial forces from Zanker and Götz (1990) (thin lines). The inertial forces clearly constitute a large fraction of the total measured forces. (C) Averaged aerodynamic forces $\left(F_{\text {aero-x }}\right.$ and $\left.F_{\text {aero-z }}\right)$ derived as the difference beween the traces shown in B. In order to test for potential errors due to inaccuracies in estimating the magnitude of the inertial forces, we used three different magnitudes of inertial forces for subtraction: $100 \%$ (thick line), $75 \%$ and $125 \%$ (thin lines) of those calculated by Zanker and Götz (1990). Varying the magnitude of the inertial forces over this range does not have substantial effects on the time course of the reconstructed aerodynamic forces. vf, ventral flip; dr, dorsal reversal.

wakes in that the ventral ends of the vortex ring do not immediately fuse above or below the body of the insect, but instead attach separately on either side of the thorax. Only after sliding along the fly's body to the tip of the abdomen do the two ends of the vortex loop fuse to form a complete ring. The pattern of vortex formation would predict that the aerodynamic forces produced by fruit flies oscillate throughout the wing stroke - with a large force corresponding with the enlargement of the vortex ring during the downstroke and ventral flip, and little force being generated during the upstroke. The results of laser interferometry partially verify this prediction. The aerodynamic forces do indeed oscillate, yet the measured forces lag behind their expected temporal position by approximately $1 \mathrm{~ms}$, corresponding to about one-fifth of the stroke cycle. In the following discussion, we will attempt to synthesize these two observations in order to present some plausible models for the mechanisms of force generation by Drosophila.

\section{Force generation during the wing stroke}

On the basis of the flow visualizations, it appears that the fly generates a vortex loop composed of the vorticity shed during the downstroke and ventral reversal. The strength of this vorticity may be estimated by relating the changes in fluid momentum to the corresponding aerodynamic forces. The momentum of a vortex ring, $M_{\mathrm{v}}$, is equal to $\rho \kappa A$, where $\rho$ is air density, $\kappa$ is ring circulation (using the nomenclature of Spedding et al. 1984) and $A$ is the area of the vortex ring (Milne-Thomson, 1966). As shown by the wake visualizations, the vortex rings of the fly enclose the area swept by the two wings within their stroke plane. Thus, we may estimate the momentum of the fly's vortex ring as:

$$
M_{\mathrm{v}}=\rho \kappa \phi R^{2}
$$

where $\phi$ is the angular position of the wing in radians and $R$ is wing length. Differentiating equation 1 with respect to time yields $F_{\mathrm{v}}$, the force resulting from the change in fluid momentum associated with a single vortex:

$$
F_{\mathrm{v}}=\frac{\mathrm{d} M_{\mathrm{v}}}{\mathrm{d} t}=\rho R^{2} \frac{\mathrm{d}(\kappa \phi)}{\mathrm{d} t} .
$$

In the simplest model of stroke kinematics, the two wings start together $[\phi(0)=0]$ with no bound circulation $[\kappa(0)=0]$. Solving for $\kappa$ under these conditions yields:

$$
\kappa(\tau)=\frac{\tau}{\phi(\tau) \rho R^{2}}\left[\frac{1}{\tau} \int_{0}^{\tau} F_{\mathrm{v}}(t) \mathrm{d} t\right] .
$$

The quantity in brackets is simply the average force, $\overline{F_{\mathrm{V}}}$, from time $t=0$ to $t=\tau$. The circulation, $\kappa_{\mathrm{s}}$, at the end of a stroke of duration $\tau_{\mathrm{s}}$ is given by:

$$
\kappa_{\mathrm{s}}=\frac{\tau_{\mathrm{s}}}{\Phi \rho R^{2}} \overline{F_{\mathrm{v}}},
$$

where $\Phi$ is the full stroke amplitude. This equation provides a means of calculating the ring circulation generated during a single stroke from the average force and simple kinematic parameters. Estimates of ring circulation based on our force measurements are given in Table 1 . On the basis of the flow visualization results, the ring circulation should correspond only to the forces generated during the downstroke and ventral

Table 1. Estimates of ring circulation and momentum based on equation 4

\begin{tabular}{lcccc}
\hline & $\begin{array}{c}\text { Stroke } \\
\text { time } \\
(\mathrm{ms})\end{array}$ & $\begin{array}{c}\text { Average } \\
\text { force } \\
(\mu \mathrm{N})\end{array}$ & $\begin{array}{c}10^{3} \times \text { ring } \\
\text { circulation, } \\
\kappa \\
\left(\mathrm{m}^{2} \mathrm{~s}^{-1}\right)\end{array}$ & $\begin{array}{c}10^{9} \times \text { ring } \\
\text { momentum, } \\
M_{\mathrm{V}} \\
\left(\mathrm{kg} \mathrm{m} \mathrm{s}^{-1}\right)\end{array}$ \\
\hline $\begin{array}{l}\text { Force model } \\
\text { average }\end{array}$ & 3.47 & 19.9 & 3.83 & 69.1 \\
$\begin{array}{l}\text { Upstroke } \\
\text { average }\end{array}$ & 1.79 & 28.2 & 2.80 & 50.5 \\
$\begin{array}{l}\text { Full stroke } \\
\text { average }\end{array}$ & 5.26 & 22.6 & 6.60 & 118.8 \\
$\begin{array}{l}\text { Average force } \\
\text { at } 24^{\circ} \text { elevation }\end{array}$ & 3.47 & 10.6 & 2.06 & 37.2 \\
\end{tabular}

The stroke durations were based on the mean wingbeat period $(5.26 \mathrm{~ms})$ and the average downstroke-upstroke duty cycle of approximately $2: 1$.

The values of wing length $(2.47 \mathrm{~mm})$ and stroke amplitude (2.46 rad) were taken from Zanker (1990a).

As explained in the text, the calculations are bracketed by using four different estimates of $\overline{F_{\mathrm{v}}}$. The calculations of average force at $24^{\circ}$ elevation are based on our measurement of the mean flight force and the work of Götz and Wandel (1984), who found that the mean force vector is invariably oriented at an angle of $24^{\circ}$ with respect to the longitudinal body axis.

The weight of a typical Drosophila melanogaster is about $10 \mu \mathrm{N}$. 
2100 M. H. DicKINSON AND K. G. GöTZ
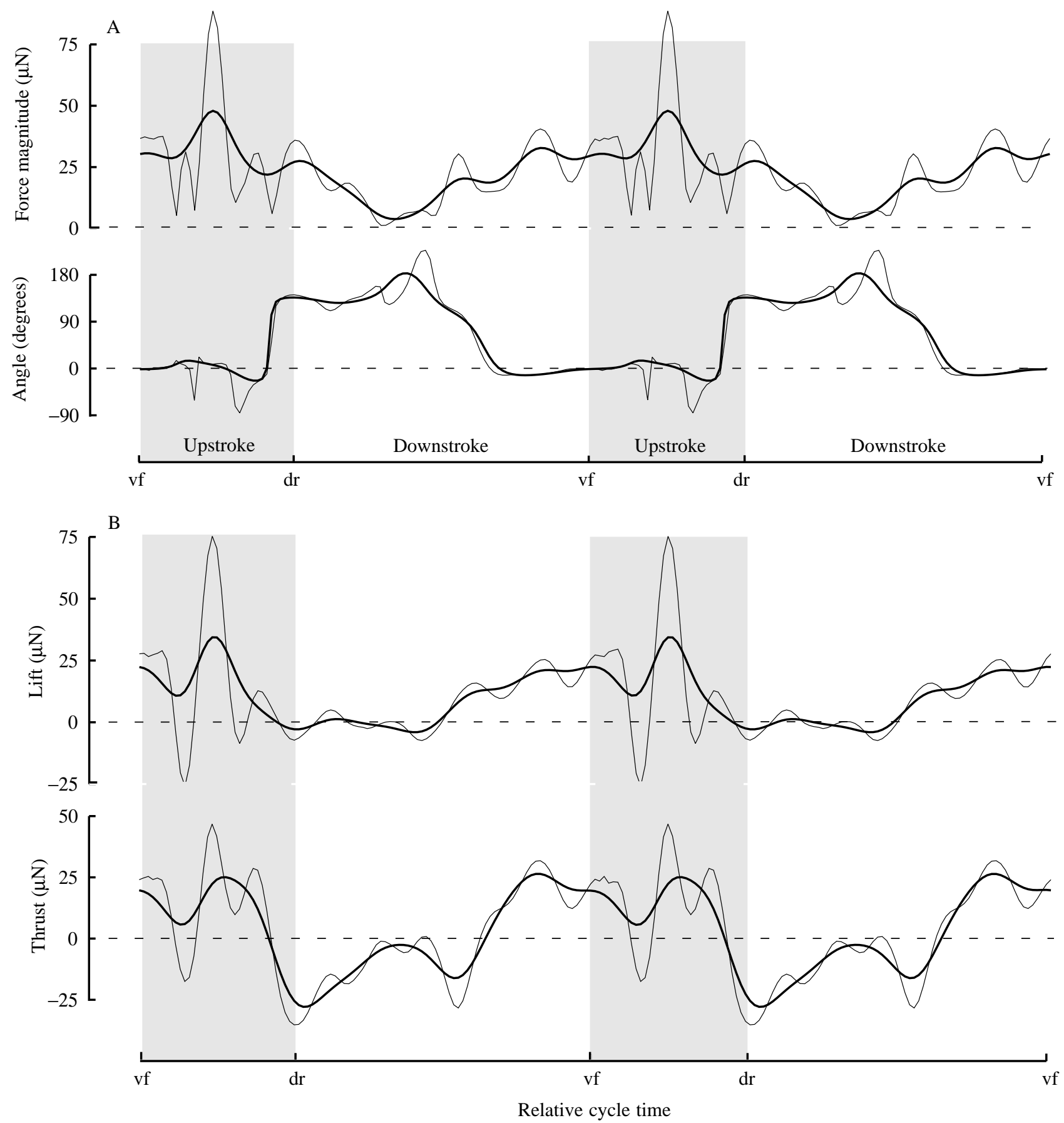

Fig. 11. Reconstruction of total aerodynamic force as well as lift and thrust vectors for a slowly flying animal. (A) The magnitude and direction of net aerodynamic force vectors were calculated trigonometrically from the $F_{\text {aero-x }}$ and $F_{\text {aero-z }}$ traces shown in Fig. $10 \mathrm{C}$ (using the $100 \%$ values of the inertial forces for subtraction). Because of potential contamination by superimposed oscillations at higher frequencies (see text), the filtered (thick line) and unfiltered (thin line) versions of each waveform have been plotted. We used a fourth-order zero phase-lag digital Butterworth filter with a cut-off frequency of $1.5 \mathrm{kHz}$. The magnitude of the flight force vector grows during the downstroke and starts to decay during the upstroke, with the peak forces oriented primarily along the axis of the body. (B) Using the same data, the lift and thrust have been reconstructed for an animal flying with a body angle of $50^{\circ}$ with respect to the horizontal, a posture expected for animals in forward flight at $20 \mathrm{~cm} \mathrm{~s}^{-1}$. As in A, both filtered and unfiltered versions of the data are shown. Both lift and thrust are positive from the late downstroke to the late upstroke. During the early part of the downstroke, lift production is zero, while thrust is negative. vf, ventral flip; dr, dorsal reversal.

flip. Averaging the force record in Fig. 11 over the downstroke interval yields a ring circulation of $3.83 \times 10^{-3} \mathrm{~m}^{2} \mathrm{~s}^{-1}$. Although inconsistent with our flow visualizations, ring circulation that would result from the average upstroke force is $2.80 \times 10^{-3} \mathrm{~m}^{2} \mathrm{~s}^{-1}$. However, because of the unexplained temporal shift in the periodic aerodynamic forces relative to 
the stroke kinematics, we cannot be confident that the reconstructed forces for either stroke correspond strictly to the intervals of vortex loop formation. Nevertheless, we can make reasonable estimates of the range of values. A liberal estimate of ring circulation would be obtained by using the average force during both the upstroke and downstroke $(22.6 \mu \mathrm{N})$ and assuming that it was generated over the entire wingbeat period. This procedure yields a ring circulation of $6.60 \times 10^{-3} \mathrm{~m}^{2} \mathrm{~s}^{-1}$. A more conservative estimate would be obtained by using the magnitude of the mean force vector $(10.6 \mu \mathrm{N})$ and assuming that it was produced entirely during the downstroke. This set of assumptions yields a ring circulation of $2.06 \times 10^{-3} \mathrm{~m}^{2} \mathrm{~s}^{-1}$. Thus, the estimated ring circulation generated by a flying fruit fly falls somewhere between $2 \times 10^{-3}$ and $7 \times 10^{-3} \mathrm{~m}^{2} \mathrm{~s}^{-1}$. At first glance, these values appear small, being roughly three orders of magnitude lower than that predicted for a slowly flying pigeon (1.79 $\mathrm{m}^{2} \mathrm{~s}^{-1}$; Spedding et al. 1994). However, given the dimensions and velocity of a Drosophila wing during flight, even a value as low as $2 \times 10^{-3} \mathrm{~m}^{2} \mathrm{~s}^{-1}$ could only be generated by aerodynamically efficient wings. The ring circulation should be roughly comparable to the bound sectional circulation of the wings. According to Kutta-Joukowski theory, the sectional lift coefficient of an airfoil is given by $2 \kappa / U c$, where $U$ is the wing velocity and $c$ is the mean chord length (circulation, $\kappa$, in the Kutta-Joukowski equation is often denoted with the symbol $\Gamma$ ). Taking values of $U=1.25 \mathrm{~m} \mathrm{~s}^{-1}$ and $c=0.85 \mathrm{~mm}$, from Zanker and Götz (1990), this predicts a lift coefficient of approximately 3.7, which would certainly require the operation of unsteady mechanisms. Such processes might include delayed stall and the presence of a leading-edge vortex (Spedding, 1992; Dickinson and Götz, 1993; Ellington, 1995). Although we saw no direct evidence for a leading-edge vortex, such a structure would probably be beyond the resolution of our visualization techniques. Ellington (1995) has succeeded in visualizing such a structure during the downstroke in Manduca sexta using a smoke rake, but only after much effort. Unlike the enormous structures that may be easily visualized on a two-dimensional model (Dickinson and Götz, 1993; Dickinson, 1994), the leading-edge vortices on an actual insect wing are likely to be small owing to the axial flow that should remove energy from the developing vortex (Maxworthy, 1979, 1981; Spedding and Maxworthy, 1986; Wu et al. 1991). On the basis of his experiments with Manduca sexta, Ellington and his colleagues (Ellington, 1995; Willmott, 1995; C. van den Berg and C. P. Ellington, in preparation) have argued that the magnitude of this length-wise flow can be quite large and may serve to stabilize the leading-edge vortices so that they remain attached for a longer translational distance. At the moment, this complicated process of dynamic stall, as it unfolds in three dimensions, is the most likely explanation for the enhanced circulation generated by the fly's wings during the downstroke.

\section{Time course of force production}

There are several possible explanations for the discrepancy between our flow visualizations and force measurements. First, it is possible that the lag in the aerodynamic forces relative to the stroke kinematics was due to a phase delay caused by the dynamic properties of the interferometric sensor. However, as mentioned in an earlier section, it should take the wire no more than $90 \mu$ s to reach within $1 \%$ of its new equilibrium value following the application of a $10 \mu \mathrm{N}$ force. Thus, the dynamics of the sensor cannot easily explain the temporal delay.

Second, the time course of the force transients might be an artifact caused by incorrect subtraction of the inertial forces required to reconstruct the aerodynamic components. As indicated in Fig. 10, varying the magnitude of the inertial force did not introduce substantial changes in the resultant time histories of the aerodynamic forces. Such inaccuracies might be responsible for differences in the magnitude of the calculated aerodynamic forces, yet under all conditions the maximum and minimum forces do not precisely align with the upstroke and downstroke. During stroke reversal, the flipping wings of Drosophila appear to rotate about their long axis. Asymmetry in the rotatory acceleration and deceleration of the wing mass on either side of this axis is inconspicuous when the wing kinematics are examined using a stroboscope. If present, such asymmetries could account for minor increments or decrements of the calculated inertial forces. The greatest effects are expected during the ventral flip and during the dorsal squeeze, clap and fling, where a reconstruction of the aerodynamic forces is particularly difficult.

The third explanation for the discrepancy between the flow visualizations and the force measurements is that the flow visualizations might not have provided sufficient temporal or spatial resolution to identify the shedding of tip vorticity during the upstroke. Some evidence for the presence of two vortex rings per stroke in the wakes of blowflies can be found in Wood's (1970), anemometric study of Calliphora erythrocephalia and Phormia regina. Wood reported that the air velocity within the wake showed two distinct peaks, which he used as evidence to claim that forces are generated during both the upstroke and the downstroke. However, close inspection of his results indicates that the majority of his records, including those that were likely to sample the air velocity in the center of the fly's wake, actually show a single velocity peak within each wing stroke. Further, because Wood flew his tethered blowflies within a $2 \mathrm{~m} \mathrm{~s}^{-1}$ air flow, his results are not directly comparable with the near-hovering conditions in the present study.

As reviewed by Ellington (1995), there is some controversy in the literature over whether or not tip vorticity is shed during the upstroke. While Brodsky's (1991, 1994) wake reconstructions from flow visualizations of butterflies include tip vorticities shed during the upstroke, Grodnitsky and Morozov (1992, 1993) did not observe comparable structures within the wakes of several insects, including bugs, flies, lacewings and two lepidopteran species. The absence of upstroke vorticity is also consistent with the patterns described for slowly flying birds (Kokshaysky, 1979; Spedding et al. 1984; Spedding, 1986) and bats (Rayner et al. 1986). Comparisons among species should be made cautiously, however, especially for fruit flies, which operate at both a higher wingbeat frequency and a lower 


\section{M. H. Dickinson AND K. G. GöTZ}

Reynolds number than any species whose wakes have been described previously. Our own visualizations support the passage of one single vortex ring during each stroke, not the linked double ring described by Brodsky (1991, 1994). It is difficult to explain how our methods could clearly identify the formation of a vortex ring during the downstroke and completely miss the formation of a comparable structure during the upstroke (Fig. 4). In any event, if the upstroke tip vorticity was too weak to be manifest by our methods, then the corresponding wing circulation is unlikely to contribute significantly to force generation. However, we did find evidence for elevated vorticity generated by wing rotation during the ventral flip (Fig. 6), which might account for the continuation of force production into the beginning of the upstroke. This vorticity produced during the flip apparently fuses with the stopping vortex filaments shed from the wing at the end of the downstroke.

The dominance of the downstroke and ventral reversal as the force-generating portions of the wing stroke makes sense given the kinematic alterations that are used by flies for the control of flight forces. During optokinetic turning responses in Drosophila, the trajectory of the downstroke changes (the wing follows a more rostral path on the outside of an intended turn), while the kinematics of the upstroke is unaltered (Zanker, 1990 b). In Calliphora vicina, the gross changes in the pattern of the wing stroke trajectory during turning responses, from a figure-of-eight to an open ellipse, are accomplished solely by alterations in the kinematics of the downstroke. Further, the two largest steering muscles in this blowfly, b1 and b2, control both wing stroke amplitude and rostral shifts in the downstroke trajectory, but have no effect on the trajectory of the upstroke (Tu and Dickinson, 1996). These results support the notion that, in some flies at least, the upstroke kinematics may have less aerodynamic significance.

One final explanation for the delay between wing motion and force production is that it represents a real fluid mechanical phenomenon. For example, at the start of the upstroke, the motion of the wings is such that the drag they encounter would have a sizable component oriented along the body axis and, thus, of the correct orientation to explain the aerodynamic forces measured at that time. According to this scheme, the wings would function to generate lift-based forces during the downstroke and drag-based forces during the upstroke. The problem with this hypothesis, however, is that except for the small component of skin friction, drag is still a pressure-based phenomenon and its presence should be manifest in the shedding of vorticity during the upstroke. Whether forces generated during the upstroke are attributed to lift or drag, the wing must generate and shed vorticity in either case. The flow visualizations, however, are not consistent with vortex shedding during the upstroke. If a fluid dynamic mechanism were responsible for the apparent presence of forces during the upstroke, it would have to be a phenomenon that did not leave behind a clear signature of vorticity in the wake.

As discussed throughout this paper, both the flow visualizations and force measurements that we have presented are subject to error. Nevertheless, given the experimental challenges associated with the small size and high wingbeat frequency of Drosophila, we may have approached the limits of resolution that are currently available. A more satisfactory explanation for the apparent phase lag between our force records and flow visualizations may eventually emerge after the development of new measurement techniques or experiments with realistic physical models.

\section{The Wagner effect and the absence of upstroke vorticity}

The absence of upstroke vorticity, if true under certain conditions, begs a question: why do the wings not develop circulation during the upstroke? This is a general question that can be applied to hovering birds and other animals that produce a single vortex with each stroke. Several factors might contribute to the absence of upstroke vorticity. First, the angle of attack of the wing with respect to the local flow might be low during the upstroke. According to the kinematic reconstruction of Zanker and Götz (1990), the angle of attack during the upstroke in Drosophila is negative and slightly smaller in magnitude than the angle of attack during the downstroke. The negative angle of attack means that the oncoming air would strike the morphological dorsal surface of the wing, which should generate an upstroke vortex ring with a similar orientation to the downstroke ring. However, this calculation of the aerodynamic angle of attack was based on a stroke-averaged estimate of the induced velocity vector. As indicated by our flow visualizations, the velocity field around the wing is time-dependent, and the instantaneous angles of attack may be somewhat different from those calculated using a constant induced velocity term. Despite these possible inaccuracies, the angle of attack of the wings during the upstroke is undoubtedly negative, and any circulation generated would have the opposite sense to that produced during the downstroke. The durations of the two strokes are also quite different, as the downstroke accounts for just less than twothirds of the entire wingbeat cycle. However, although this would mean that vorticity has less time to develop, the wing must travel the same distance during the upstroke and accomplishes this by traveling at a greater velocity. If anything, one would expect this increased velocity to result in greater circulation during the upstroke. Thus, neither differences in angle of attack nor stroke duty cycle can easily explain the apparent absence of upstroke circulation in Drosophila.

The most likely explanation for the absence of, or reduction in, circulation lies with the peculiar flow conditions at the start of the upstroke. In Drosophila, the downstroke begins with the intensely studied 'squeeze, clap and fling' motion (Weis-Fogh, 1973; Bennett, 1977; Maxworthy, 1979; Ellington, 1984b; Spedding and Maxworthy, 1986; Götz, 1987). The close apposition of the wing tips during this behavior serves to link the fluid mechanical processes of the two wings so that the bound vorticity of one wing constitutes the starting vortex of the other. As the wings separate, their tip vortices fuse and thus create the dorsal segment of the developing vortex loop. These conditions of close proximity are not repeated at the beginning of the upstroke, at which time the wings are separated by 

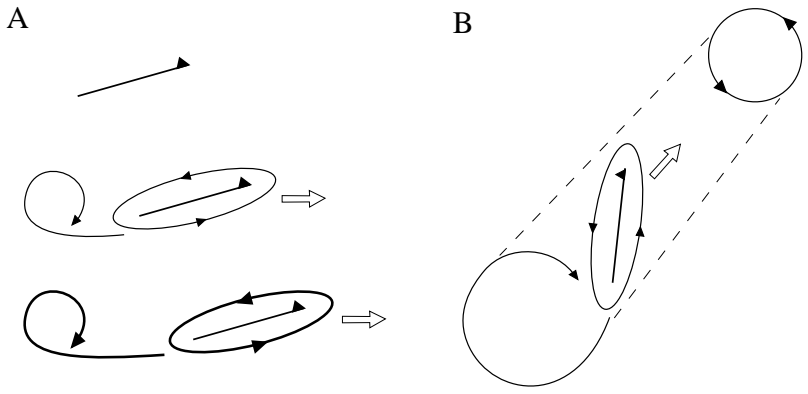

Fig. 12. Model for the attenuation of upstroke circulation according to the Wagner effect shown in a schematized sagittal view. The open arrow shows the direction of wing motion. (A) The Wagner effect as described for a wing started impulsively from rest. As the wing starts to move, the bound circulation is in close proximity with the free starting vortex, which possesses the opposite sense. The full steadystate circulation is not attained until the wing moves a sufficient distance from the starting vortex. The strength of circulation is indicated by line thickness. (B) At the start of the upstroke, the Wagner effect is magnified by the presence of the shed vorticity of the downstroke, which possesses the same rotational direction as the starting vortex. Under these conditions, the wing must translate even further before it can attain high values of circulation. The dashed lines mark the outline of the downstroke vortex loop.

approximately $80^{\circ}$. This separation is too large for the exchange of vorticity, as shown by the lack of fusion between the shed vortex filaments after the ventral flip. At the start of the upstroke, two factors might inhibit the generation of circulation by the wings. First, their separation does not allow the swapping of starting and bound vorticity that operates at the start of the downstroke. Second, at the start of the upstroke, the wings begin translating in the presence of the large filaments shed during stroke reversal. These filaments consist of the stopping vorticity of the downstroke augmented by the shed rotational circulation generated during the ventral flip. This free vorticity would have the same sense as that of the starting vortex of the upstroke. The Wagner effect (Wagner, 1925) describes how the bound circulation of a wing starting from rest is delayed by the presence of its own starting vortex. In the case of fly wings at the start of the upstroke, this effect will be greatly amplified because each wing must separate not only from its own starting vortex but also from the large shed vortex of the previous stroke. This 'additive-Wagner effect' (Fig. 12) would greatly attenuate the development of bound circulation and could explain the absence of tip vortices and force generation during the upstroke. Evidence for such an effect may be seen in previous studies on unsteady aerodynamics mechanisms in two-dimensional model Drosophila wings (Dickinson and Götz, 1993; Dickinson, 1994). The generation of lift by the model was greatly attenuated when translation was preceded by a two-chord downstroke and rotation (Fig. 7A in Dickinson, 1994). When following a previous stroke reversal, the lift generated after two chords of travel at an angle of attack of $36^{\circ}$ was nearly 3.5 times lower than that generated during an impulsive start from rest. This attenuation was critically dependent on the duration of the previous stroke, indicating that the sign of the attached vorticity shed by the previous stroke was an essential determinant of the effect's magnitude. After two chords of translation at a high angle of attack, the initial leading-edge vortex was still attached to the model wing and, after shedding, had the correct rotational sense to inhibit the development of circulation in the subsequent stroke via the additive-Wagner effect. The attenuation is not present following a 7.5 chord downstroke, in which the leading-edge vortex has detached before rotation, replaced by a trailing-edge vortex of opposite sense.

The negative influence of one stroke's circulation on the next is probably not exclusive to flies or other animals that perform a clap and fling. Rather, the proposed additive-Wagner effect might influence the design of kinematics in many animals that employ a reciprocating wing stroke. One consequence of the effect is that it may not be profitable to produce force during both the upstroke and downstroke, since the presence of circulation in one half-stroke will delay the generation of circulation in the next. Instead, the animal might do better by using one single power stroke, alternating with a noncirculatory recovery stroke. The lack of circulation during the recovery stroke ensures more rapid force generation during the power stroke. The effect might also explain the transition from the single vortex loop pattern seen in hovering animals to the more complicated linked vortex chains described by Brodsky (1991, 1994). As the advance ratio increases, the vorticity shed during the downstroke and ventral flip will move rearwards at an increasing rate relative to the velocity of the wings. At a certain point, the rearward velocity of the vortex loop will be fast enough so that the shed downstroke vorticity is far enough away from the wing to allow the formation of upstroke circulation. Under these conditions, the wake would take on the 'linked-chain' structure described by Brodsky (1991, 1994), in which the tip vortex filaments of each stroke are connected by common starting-stopping vortices at stroke reversal. In addition to the advance ratio, the transition from a discrete series of vortex rings to a linked chain would depend on many factors, including Reynolds number, wing separation and wing shape, all of which may contribute to the discrepancies already described in the flow visualizations of various insects.

We wish to thank Michael Renner for his extensive help with the acquisition and processing of the flight force measurements. Fritz-Olaflehmann and Roland Strauss also provided additional technical support during the execution of this work. The authors would also like to thank the many helpful and provocative comments of two anonymous reviewers. This work was funded in part by the Max Planck Gesellschaft, the David and Lucille Packard Foundation and NSF Grant IBN-9208765 to M.H.D.

\section{References}

BENNETT, L. (1977). Clap and fling aerodynamics - an experimental evaluation. J. exp. Biol. 69, 261-272. 


\section{M. H. Dickinson AND K. G. GöTZ}

BRODSKY, A. K. (1991). Vortex formation in the tethered flight of the peacock butterfly Inachis io L. (Lepidoptera, Nymphalidae) and some aspects of insect flight evolution. J. exp. Biol. 161, 77-95.

Brodsky, A. K. (1994). The Evolution of Insect Flight. New York: Oxford University Press.

BuckHOLZ, R. H. (1981). Measurements of unsteady periodic forces generated by the blowfly flying in a wind tunnel. J. exp. Biol. 90, 163-173.

Cheer, A. Y. L. And Koehl, M. A. R. (1987). Paddles and rakes: Fluid flow through bristled appendages of small organisms. $J$. theor. Biol. 129, 17-39.

Cloupeau, M., Devillers, J. F. and Devezeaux, D. (1979). Direct measurements of instantaneous lift in desert locust: comparison with Jensen's experiments on detached wings. J. exp. Biol. 80, 1-15.

DAVID, C. T. (1978). The relationship between body angle and flight speed in free-flying Drosophila. Physiol. Ent. 3, 191-195.

Dickinson, M. H. (1994). The effects of wing rotation on unsteady aerodynamic performance at low Reynolds numbers. J. exp. Biol. 192, 179-206.

Dickinson, M. H. AND GöTZ, K. G. (1993). Unsteady aerodynamic performance of model wings at low Reynolds numbers. J. exp. Biol. 174, 45-64.

Dickinson, M. H., Lehmann, F.-O. And GötZ, K. G. (1993). The active control of wing rotation by Drosophila. J. exp. Biol. 182, 173-189.

ElLington, C. P. (1984a). The aerodynamics of hovering flight. III. Kinematics. Phil. Trans. R. Soc. Lond. B 305, 41-78.

Ellington, C. P. (1984b). The aerodynamics of hovering flight. IV. Aerodynamic mechanisms. Phil. Trans. R. Soc. Lond. B 305, 79-113.

Ellington, C. P. (1984c). The aerodynamics of hovering flight. V. A vortex theory. Phil. Trans. R. Soc. Lond. B 305, 115-144.

Ellington, C. P. (1995). Unsteady aerodynamics of insect flight. In Biological Fluid Dynamics (ed. C. P. Ellington and T. J. Pedley). Symp. Soc. exp. Biol. 49, 109-129. Cambridge: The Company of Biologists Ltd.

GöTZ, K. G. (1968). Flight control in Drosophila by visual perception of motion. Kybernetik 4, 199-208.

Götz, K. G. (1987). Course-control, metabolism and wing interference during ultralong tethered flight in Drosophila melanogaster. J. exp. Biol. 128, 35-46.

GöTZ, K. G. AND WANDEL, U. (1984). Optomotor control of the force of flight in Drosophila and Musca. II. Covarience of lift and thrust in still air. Biol. Cybernetics 51, 135-139.

GrodNitsky, D. L. AND Morozov, P. P. (1992). Flow visualization experiments on terthered flying green lacewings Chrysopa dasyptera. J. exp. Biol. 169, 143-163.

GrodNitsKy, D. L. AND Morozov, P. P. (1993). Vortex formation during tethered flight of functionally and morphologically twowinged insects, including evolutionary considerations on insect flight. J. exp. Biol. 182, 11-40.

JEnsEn, M. (1956). Biology and physics of locust flight. III. The aerodynamics of locust flight. Phil. Trans. R. Soc. Lond. B 239, 511-552.

KOKSHAYSKY, N. V. (1979). Tracing the wake of a flying bird. Nature 279, 146-148.

LEHMANN, F.-O. (1994). Aerodynamische, kinematische und electrophysiologische Aspekte der Flugkrafterzeugung und Flugkraftsteuerung bei der Taufliege Drosophila melanogaster. Dissertation, Eberhad-Karls-Universität Tübingen.

MAXWORTHY, T. (1979). Experiments on the Weis-Fogh mechanism of lift generation by insects in hovering flight. Part 1. Dynamics of the 'fling'. J. Fluid Mech. 93, 47-63.

MaXworthy, T. (1981). The fluid dynamics of insect flight. J. Fluid Mech. 93, 47-63.

Milne-Thomson, L. M. (1966). Theoretical Aerodynamics. New York: Macmillan.

RAYNER, J. M. V. (1979a). A new approach to animal flight mechanics. J. exp. Biol. 80, 17-54.

Rayner, J. M. V. (1979b). A vortex theory of animal flight. Part 1. The vortex wake of a hovering animal. J. Fluid Mech. 91, 697-730.

RAYNER, J. M. V., Jones, G. AND Thomas, A. (1986). Vortex flow visualizations reveal change in upstroke function with flight speed in bats. Nature 321, 162-164.

SPEDDING, G. R. (1986). The wake of a jackdaw (Corvus mondela) in slow flight. J. exp. Biol. 125, 287-307.

SPEDDING, G. R. (1987). The wake of a kestrel (Falco tinnunculus) in flapping flight. J. exp. Biol. 127, 59-78.

SPEDDING, G. R. (1992). The aerodynamics of insect flight. In Advances in Comparative and Environmental Physiology (ed. R. McN. Alexander), pp. 51-111. London: Springer-Verlag.

SPedding, G. R. AND MaXworthy, T. (1986). The generation of circulation and lift in a rigid two-dimensional fling. J. Fluid Mech. 165, 247-272.

Spedding, G. R., Rayner, J. M. V. And Pennycuick, C. J. (1984). Momentum and energy in the wake of a pigeon (Columba livia) in slow flight. J. exp. Biol. 111, 81-102.

Tu, M. S. AND Dickinson, M. H. (1996). The control of wing kinematics by two steering muscles of the blowfly, Calliphora vicina. J. comp. Physiol. A (in press).

WAGNER, H. (1925). Über die Entstehung des dynamischen Äuftriebes von Tragflügeln. Z. angew. Math. Mech. 5, 17-35.

WeIs-Fogh, T. (1973). Quick estimates of flight fitness in hovering animals, including novel mechanisms for lift production. J. exp. Biol. 59, 169-230.

WILKIN, P. J. (1990). The instantaneous force on a desert locust, Schistocerca gregaria (Orthoptera: Acrididae), flying in a wing tunnel. J. Kansas ent. Soc. 63, 316-328.

WILKIN, P. J. (1991). Instantaneous aerodynamic forces developed by an Indian moon moth, Actias selene, in near hovering flight. Physiol. Zool. 64, 193-211.

Wilkin, P. J. AND Williams, M. H. (1993). Comparison of the instantaneous aerodynamic forces on a sphingid moth with those predicted by quasi-steady aerodynamic theory. Physiol. Zool. 66, 1015-1044.

WillmotT, A. P. (1995). The mechanics of hawkmoth flight. Doctoral thesis, Cambridge University.

Wolf, H. (1993). The locust tegula: significance for flight rhythm generation, wing movement control and aerodynamic force production. J. exp. Biol. 182, 229-253.

Wood, J. (1970). A study of the instantaneous air velocities in a plane behind the wings of certain Diptera flying in a wind tunnel. J. exp. Biol. 52, 17-25.

Wu, J. Z., VakiLI, A. D. AND Wu, J. M. (1991). Review of the physics of enhancing vortex lift by unsteady excitation. Progr. aero. Sci. 28, 73-131.

ZANKER, J. M. (1990a). The wing beat of Drosophila melanogaster. I. Kinematics. Phil. Trans. R. Soc. Lond. B 327, 1-18.

ZANKER, J. M. (1990b). The wing beat of Drosophila melanogaster. III. Control. Phil. Trans. R. Soc. Lond. B 327, 45-64.

ZANKER, J. M. AND GÖTZ, K. G. (1990). The wing beat of Drosophila melanogaster. II. Dynamics. Phil. Trans. R. Soc. Lond. B 327, 19-44. 